\title{
STABILITY AND BIFURCATION IN A SIMPLIFIED FOUR-NEURON BAM NEURAL NETWORK WITH MULTIPLE DELAYS
}

\author{
XIANG-PING YAN AND WAN-TONG LI
}

Received 23 May 2005; Accepted 4 September 2005

We first study the distribution of the zeros of a fourth-degree exponential polynomial. Then we apply the obtained results to a simplified bidirectional associated memory (BAM) neural network with four neurons and multiple time delays. By taking the sum of the delays as the bifurcation parameter, it is shown that under certain assumptions the steady state is absolutely stable. Under another set of conditions, there are some critical values of the delay, when the delay crosses these critical values, the Hopf bifurcation occurs. Furthermore, some explicit formulae determining the stability and the direction of periodic solutions bifurcating from Hopf bifurcations are obtained by applying the normal form theory and center manifold reduction. Numerical simulations supporting the theoretical analysis are also included.

Copyright (c) 2006 X.-P. Yan and W.-T. Li. This is an open access article distributed under the Creative Commons Attribution License, which permits unrestricted use, distribution, and reproduction in any medium, provided the original work is properly cited.

\section{Introduction}

In recent years, the interest in investigating the dynamics of neural networks has been steadily increasing since Hopfield [11] constructed a simplified neural network model. Based on the Hopfield neural network, Marcus and Westervelt [14] argued that time delays always appear in the signal transmission and therefore they proposed a neural network model with delays. Hereafter, various dynamical models modeling delayed neural networks have been proposed and investigated widely to understand the dynamics behavior of the like-neurons (see $[1,2,6,7]$ ) and there has been an extensive literature on the local and global stability analysis of the delayed neural networks (see $[6,15,19,23]$ and the references cited therein). It is well known that the study on dynamical systems not only involve a discussion of stability, but also involve many dynamical behaviors such as periodic phenomenon, bifurcation and chaos. In particular, the properties of periodic solutions are of great interest, which can arise through the Hopf bifurcation in delayed systems, see Hale [8], Liu and Yuan [13], Wei and Ruan [20] and Wei and Velarde [21].

Hindawi Publishing Corporation

Discrete Dynamics in Nature and Society

Volume 2006, Article ID 32529, Pages 1-29

DOI 10.1155/DDNS/2006/32529 
As large-scale nonlinear dynamical systems, neural networks are complex while the dynamics of the delayed neural networks are even richer and more complicated. Neural networks with delays can exhibit very rich dynamics [22]. Since an exhaustive analysis of the dynamics of large-scale dynamical systems is quite difficult, Babcock and Westervelt [1] suggested examining carefully the dynamical behaviors of the simple and lower-order networks. Consequently, in recent years, there has been an extensive literature about the studies of the dynamics of some special and simple lower-order neural networks (see $[3-5,7,16,17,20,22])$. However, the studies of the dynamics regarding higher-order systems modelling the neural networks with more neurons and multiple delays are rare. To the best of our knowledge, there is a few literatures have investigated higher-order neural networks (see $[18,21]$ for the case of three neurons and multiple delays and $[4,12]$ for a ring of four neurons). In fact, in practical applications, the neural networks with more neurons and multiple delays are of great importance and frequency. Therefore, it is necessary to investigative the dynamical behaviors of this kind of networks.

In general, in the investigating on a delay model, linearization of the system at its steady state gives us a transcendental characteristic equation or called an exponential polynomial equation. It is well known that the steady state is stable if all eigenvalues of the corresponding transcendental characteristic equation have negative real parts, and unstable if at least one root has positive real part. Thus, a Hopf bifurcation occurs when the real part of a certain eigenvalue changes from negative to zero and to positive (i.e., the steady state changes from stability to instability). This is usually caused by the delays. However, there is a strong possibility that if the coefficients of the exponential polynomial satisfy certain assumptions, then the real parts of all eigenvalues remain negative for all values of the delay; that is, independent of the delay. The corresponding delay system is called absolutely stable (see, e.g., Hale et al. [9]). A general result in Hale et al. [9] says that a delay system is absolutely stable if and only if the corresponding ODE system is asymptotically stable and the characteristic equation has no purely imaginary roots. Therefore, a first important step in the study of the dynamics of a delayed model is to analyze in detail the distribution of zeros of the associated characteristic equation. However, most aforementioned works on the neural network focused mainly on the second-dimensional case and hence the associated characteristic equation is also second-degree exponential polynomial equation. Thus, the second-degree exponential polynomial equation has been investigated widely and exhaustively (see [20] and the cited reference therein). As far as the study of third and fourth-degree exponential polynomial equation, there are also a few literature (see, e.g., $[17,18]$ for the case of the third degree and $[4,12]$ for the case of the fourth degree).

Among a great deal of artificial neural networks, there exist a class of important twolayer heteroassociative networks, called bidirectional associative memory (BAM) neural networks with axonal signal transmission delays, has been proposed and applied in many fields such as pattern recognition and automatic control (see [6]). If there is only one neuron on the $I$-layer and three neurons on the $J$-layer, the time delay from the $I$-layer to another $J$-layer is $\tau_{1}$ while the delay from the $J$-layer back to the $I$-layer is $\tau_{2}$, and the activation functions are $f_{l}(l=2,3,4)$ (see Figure 1.1). Then the network model can be described by the following delayed differential equations, namely, functional differential 


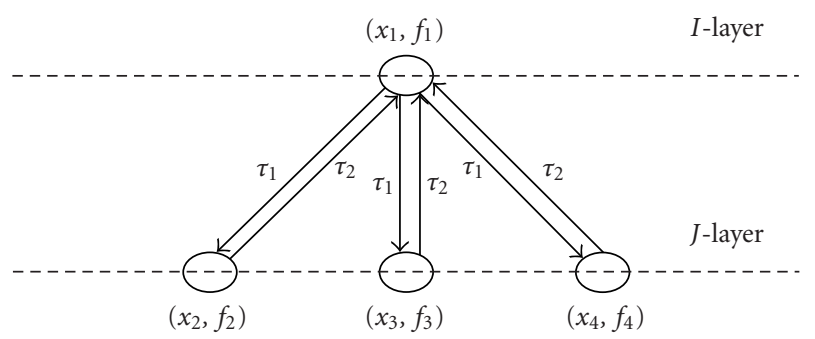

Figure 1.1. Graph of the architecture of the model (1.1).

equations

$$
\begin{aligned}
\dot{x}_{1}(t)=-\mu_{1} x_{1}(t)+ & c_{21} f_{1}\left(x_{2}\left(t-\tau_{2}\right)\right)+c_{31} f_{1}\left(x_{3}\left(t-\tau_{2}\right)\right)+c_{41} f_{1}\left(x_{4}\left(t-\tau_{2}\right)\right), \\
\dot{x}_{2}(t) & =-\mu_{2} x_{2}(t)+c_{12} f_{2}\left(x_{1}\left(t-\tau_{1}\right)\right), \\
\dot{x}_{3}(t) & =-\mu_{3} x_{3}(t)+c_{13} f_{3}\left(x_{1}\left(t-\tau_{1}\right)\right), \\
\dot{x}_{4}(t) & =-\mu_{4} x_{4}(t)+c_{14} f_{4}\left(x_{1}\left(t-\tau_{1}\right)\right),
\end{aligned}
$$

where $x_{k}(k=1,2,3,4)$ denote the state of the $k$ th neuron; $\mu_{k}>0(k=1,2,3,4)$ describe respectively the stability of internal neuron processes on the $I$-layer and the $J$-layer; the real constants $c_{l 1}(l=2,3,4)$ and $c_{1 k}(k=2,3,4)$ denote the connected weights through the neurons in two layers: the $I$-layer and the $J$-layer.

The linearization of the system (1.1) at its steady state leads to a transcendental characteristic equation in the form of

$$
\lambda^{4}+a_{3} \lambda^{3}+a_{2} \lambda^{2}+a_{1} \lambda+a_{0}+\left(b_{2} \lambda^{2}+b_{1} \lambda+b_{0}\right) e^{-\lambda \tau}=0
$$

which is a fourth-degree exponential polynomial equation.

If $b_{1}=b_{2}=0$, then (1.1) reduces to

$$
\lambda^{4}+a_{3} \lambda^{3}+a_{2} \lambda^{2}+a_{1} \lambda+a_{0}+b_{0} e^{-\lambda \tau}=0,
$$

which was investigated by Li and Wei [12], and applied the obtained results to a ring of neural network model consisting of four different neurons with instantaneous selfconnection and multiple delays, see Figure 1.2.

Clearly, if $b_{1}^{2}+b_{2}^{2} \neq 0$, then the results obtained by Li and Wei [12] fail to (1.2). Therefore, in order to study the dynamics of the neural network (1.1), it is necessary to investigate further the fourth-degree exponential polynomial equation (1.2) with $b_{1}^{2}+b_{2}^{2} \neq 0$.

In this paper, we first study the distribution of the roots of (1.2) and find that there are the following two possibilities.

(a) Under certain assumptions on the coefficients, all roots of (1.2) have negative real parts for all delay value $\tau \geq 0$.

(b) If the assumptions in (a) are not satisfied, then there is a critical value $\tau_{0}$. When the delay $\tau<\tau_{0}$, the real parts of all roots of (1.2) are still negative; when $\tau=\tau_{0}$, there is 


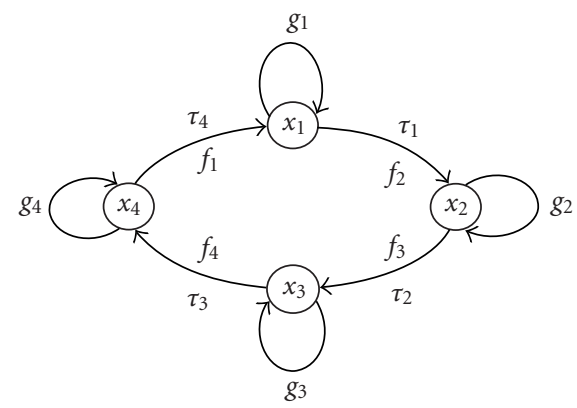

Figure 1.2. A four-neuron ring with delays and self-connection.

a pair of purely imaginary roots and all other roots have negative real parts; when $\tau>\tau_{0}$, there is at least one eigenvalue which has positive real part.

By regarding the sum $\tau$ of the two delays $\tau_{1}$ and $\tau_{2}$ as a bifurcation parameter, applying the obtained results to the BAM neural network (1.2), we show that under a set of assumptions on the coefficients, the steady state is absolutely stable (i.e., asymptotically stable independent of the delay $\tau$ ). Under another set of conditions, the steady state is conditionally stable; that is, there is a sequence of critical delay values $\tau_{0}<\tau_{1}<\ldots$, and the steady state is asymptotically stable when $\tau<\tau_{0}$, loses its stability when $\tau=\tau_{0}$, and becomes unstable when $\tau>\tau_{0}$. Thus, a Hopf bifurcation occurs at the steady state when $\tau$ passes through the critical values $\tau_{j}(j=0,1, \ldots)$.

This paper is organized as follows. In the next section, we will analyze in detail the distribution of roots of the fourth exponential polynomial equation (1.1). In Section 3, we apply the results obtained in Section 2 to (1.2), the absolute stability, conditional stability of zero equilibrium and the existence of Hopf bifurcation are studied. In Section 4, based on the normal form theory and the center manifold argument introduced by Hassard et al. [10], we derive the formulae determining the direction, stability and the period of the bifurcating periodic solution. In Section 5, by applying the results obtained in Section 4, we give a result for a special case of (1.1) determining the direction, stability and the period of the bifurcating periodic solution. Finally, to verify the theoretic analysis, a conclusion is also drawn in the end.

\section{The fourth-degree transcendental polynomial equation}

In this section, we will study in detail the distribution of zeros of the fourth-degree transcendental polynomial equation (1.2).

Consider the following fourth-degree transcendental polynomial equation

$$
\lambda^{4}+a_{3} \lambda^{3}+a_{2} \lambda^{2}+a_{1} \lambda+a_{0}+\left(b_{2} \lambda^{2}+b_{1} \lambda+b_{0}\right) e^{-\lambda \tau}=0
$$

where $a_{k}, b_{l} \in \mathbb{R}(k=0,1,2,3 ; l=0,1,2)$ are all real constants and $\sum_{l=0}^{2} b_{l}^{2} \neq 0$. Clearly, $i \omega(\omega>0)$ is a root of $(2.1)$ if and only if $\omega$ satisfies the following equation

$$
\omega^{4}-i a_{3} \omega^{3}-a_{2} \omega^{2}+i a_{1} \omega+a_{0}+\left(-b_{2} \omega^{2}+i b_{1} \omega+b_{0}\right)(\cos \omega \tau-i \sin \omega \tau)=0 .
$$


Separating the real and imaginary parts of the above equation yields the following equations

$$
\begin{aligned}
& \omega^{4}-a_{2} \omega^{2}+a_{0}=\left(b_{2} \omega^{2}-b_{0}\right) \cos \omega \tau-b_{1} \omega \sin \omega \tau \\
& -a_{3} \omega^{3}+a_{1} \omega=-\left(b_{2} \omega^{2}-b_{0}\right) \sin \omega \tau-b_{1} \omega \cos \omega \tau .
\end{aligned}
$$

Adding up the squares of the corresponding sides of the above equations leads to

$$
\begin{aligned}
\omega^{8}+ & \left(a_{3}^{2}-2 a_{2}\right) \omega^{6}+\left(2 a_{0}+a_{2}^{2}-2 a_{1} a_{3}-b_{2}^{2}\right) \omega^{4} \\
& +\left(a_{1}^{2}-2 a_{0} a_{2}+2 b_{0} b_{2}-b_{1}^{2}\right) \omega^{2}+a_{0}^{2}-b_{0}^{2}=0 .
\end{aligned}
$$

Let $z=\omega^{2}$ and denote

$$
\begin{aligned}
& a=a_{3}^{2}-2 a_{2}, \quad b=2 a_{0}+a_{2}^{2}-2 a_{1} a_{3}-b_{2}^{2}, \\
& c=a_{1}^{2}-2 a_{0} a_{2}+2 b_{0} b_{2}-b_{1}^{2}, \quad d=a_{0}^{2}-b_{0}^{2} .
\end{aligned}
$$

Then, (2.4) can be denoted simply as the following equation:

$$
z^{4}+a z^{3}+b z^{2}+c z+d=0 .
$$

Let

$$
h(z)=z^{4}+a z^{3}+b z^{2}+c z+d .
$$

Noting that $\lim _{z \rightarrow+\infty} h(z)=+\infty$, we conclude that if $d<0$, then (2.6) has at least one positive root.

From (2.7), we have

$$
\frac{d h(z)}{d z}=4 z^{3}+3 a z^{2}+2 b z+c=4 f(z)
$$

where $f(z)=z^{3}+(3 / 4) a z^{2}+(1 / 2) b z+(1 / 4) c$. Let

$$
p=\frac{8 b-3 a^{2}}{16}, \quad q=\frac{a^{3}-4 a b+8 c}{32}, \quad D_{0}=\frac{q^{2}}{4}+\frac{p^{3}}{27} \text {. }
$$

Suppose that $D_{0}>0$, then from the Cardano's formulae for the third-degree algebra equation, we know that the equation $f(z)=0$ has only one real root

$$
z_{1}^{*}=-\frac{a}{4}+\sqrt[3]{-\frac{q}{2}+\sqrt{D_{0}}}+\sqrt[3]{-\frac{q}{2}-\sqrt{D_{0}}}
$$

Noticing that $\lim _{z \rightarrow \pm \infty} h(z)=+\infty$, thus we know that $z_{1}^{*}$ is a unique minimum value point of $h(z)$ on $\mathbb{R}$. Therefore, if $d \geq 0$ and $z_{1}^{*} \leq 0$, then (2.6) has no positive roots; if $d \geq 0, z_{1}^{*}>0$ and $h\left(z_{1}^{*}\right)<0$, then (2.6) has at least one positive roots.

Assume that $D_{0}=0$, then in this case the equation $f(z)=0$ has three real roots

$$
z_{1}=-\frac{a}{4}-2 \sqrt[3]{\frac{q}{2}}, \quad z_{2}=z_{3}=-\frac{a}{4}+\sqrt[3]{\frac{q}{2}}
$$


Let

$$
z_{2}^{*}=\max \left\{-\frac{a}{4}-2 \sqrt[3]{\frac{q}{2}},-\frac{a}{4}+\sqrt[3]{\frac{q}{2}}\right\},
$$

it is easy to know that $h(z)$ is strictly monotonously increasing when $z>z_{2}^{*}$. Therefore, if $d \geq 0$ and $z_{2}^{*} \leq 0$, then (2.6) has no positive roots; if $d \geq 0, z_{2}^{*}>0$ and $h\left(z_{2}^{*}\right)<0$, then (2.6) has at least one positive root.

If $D_{0}<0$, then we know that the equation $f(z)=0$ has three real roots

$$
r_{1}^{*}=-\frac{a}{4}+2 \operatorname{Re}\{\alpha\}, \quad r_{2}^{*}=-\frac{a}{4}+2 \operatorname{Re}\{\alpha \varepsilon\}, \quad r_{3}^{*}=-\frac{a}{4}+2 \operatorname{Re}\{\alpha \bar{\varepsilon}\},
$$

where $\alpha$ is one of cubic roots of the complex number $-q / 2+\sqrt{D_{0}}$ and $\varepsilon=-1 / 2+(\sqrt{3} / 2) i$. Let $z_{3}^{*}=\max \left\{r_{1}^{*}, r_{2}^{*}, r_{3}^{*}\right\}$, we can see that if $d \geq 0$ and $z_{3}^{*} \leq 0$, then (2.6) has no positive roots; if $d \geq 0, z_{3}^{*}>0$ and $h\left(z_{3}^{*}\right)<0$, then (2.6) has at least one positive root.

Summarizing these remarks, we have the following result.

Lemma 2.1. (i) If $d<0$, then (2.6) has at least one positive root.

(ii) Suppose that $d \geq 0$, then (2.6) has no positive root if one of the following conditions holds:

(1) $D_{0}>0$ and $z_{1}^{*}<0$;

(2) $D_{0}=0$ and $z_{2}^{*}<0$;

(3) $D_{0}<0$ and $z_{3}^{*}<0$.

(iii) Suppose that $d \geq 0$, then (2.6) has at least a positive root if one of the following conditions holds:

(1) $D_{0}>0, z_{1}^{*}>0$ and $h\left(z_{1}^{*}\right)<0$;

(2) $D_{0}=0, z_{2}^{*}>0$ and $h\left(z_{2}^{*}\right)<0$;

(3) $D_{0}<0, z_{3}^{*}>0$ and $h\left(z_{3}^{*}\right)<0$.

Suppose now that (2.6) has positive roots. Without loss of generality, we may assume that (2.6) has four positive roots denoted respectively as $z_{1}, z_{2}, z_{3}$ and $z_{4}$. Then (2.4) has four positive roots $\omega_{k}=\sqrt{z_{k}}(k=1,2,3,4)$. In view of $(2.3)$, we can get

$$
\cos \omega_{k} \tau=\frac{\Delta_{1}}{\Delta}
$$

where

$$
\begin{gathered}
\Delta_{1}=b_{2} \omega_{k}^{6}+\left(a_{3} b_{1}-a_{2} b_{2}-b_{0}\right) \omega_{k}^{4}+\left(a_{0} b_{2}+a_{2} b_{0}-a_{1} b_{1}\right) \omega_{k}^{2}-a_{0} b_{0}, \\
\Delta=b_{2}^{2} \omega_{k}^{4}+\left(b_{1}^{2}-2 b_{0} b_{2}\right) \omega_{k}^{2}+b_{0}^{2} .
\end{gathered}
$$

Therefore, if we define

$$
\tau_{j}^{(k)}=\frac{1}{\omega_{k}}\left[\arccos \left(\frac{\Delta_{1}}{\Delta}\right)+2 j \pi\right], \quad k=1,2,3,4, j=0,1,2, \ldots,
$$

then (2.1) with $\tau=\tau_{j}^{(k)}$ has a pair of purely imaginary roots $\pm i \omega_{k}$. Let

$$
\tau_{0}=\tau_{0}^{\left(k_{0}\right)}=\min _{k \in\{1,2,3,4\}}\left\{\tau_{0}^{(k)}\right\}, \quad \omega_{0}=\omega_{k_{0}} .
$$


In what follows, we use a result due to Ruan and Wei [17] to analyze (2.1), which is stated as follows.

Lemma 2.2. Consider the exponential polynomial

$$
\begin{aligned}
P\left(\lambda, e^{-\lambda \tau_{1}}, e^{-\lambda \tau_{2}}, \ldots, e^{-\lambda \tau_{m}}\right)=\lambda^{n}+ & p_{1}^{(0)} \lambda^{n-1}+\cdots+p_{n-1}^{(0)} \lambda+p_{n}^{(0)} \\
+ & {\left[p_{1}^{(1)} \lambda^{n-1}+\cdots+p_{n-1}^{(1)} \lambda+p_{n}^{(1)}\right] e^{-\lambda \tau_{1}} } \\
& +\cdots+\left[p_{1}^{(m)} \lambda^{n-1}+\cdots+p_{n-1}^{(m)} \lambda+p_{n}^{(m)}\right] e^{-\lambda \tau_{m}},
\end{aligned}
$$

where $\tau_{k} \geq 0(k=1,2, \ldots, m)$ and $p_{j}^{(k)}(k=0,1,2, \ldots, m, j=1,2, \ldots, n)$ are real constants. As $\left(\tau_{1}, \tau_{2}, \ldots, \tau_{m}\right)$ vary, the sum of the order of the zeros of $P\left(\lambda, e^{-\lambda \tau_{1}}, e^{-\lambda \tau_{2}}, \ldots, e^{-\lambda \tau_{m}}\right)$ on the open right half-plane can change only if a zero appears on or crosses the imaginary axis.

Note that when $\tau=0,(2.1)$ becomes the following fourth-degree algebra equation

$$
\lambda^{4}+a_{3} \lambda^{3}+\left(a_{2}+b_{2}\right) \lambda^{2}+\left(a_{1}+b_{1}\right) \lambda+a_{0}+b_{0}=0
$$

It is easily to get the following result regarding the distribution of roots of the exponential polynomial equation (2.1) by using Lemmas 2.1 and 2.2.

Lemмa 2.3. For the exponential polynomial equation (2.1), the following states are true.

(i) If $d \geq 0$ and one of the following conditions holds,

(1) $D_{0}>0$ and $z_{1}^{*}<0$;

(2) $D_{0}=0$ and $z_{2}^{*}<0$;

(3) $D_{0}<0$ and $z_{3}^{*}<0$,

then the order of all roots with positive real parts of (2.1) has the same sum as that of all roots with positive real parts of (2.19) for all $\tau \geq 0$.

(ii) If $d<0$ or $d \geq 0$ and one of the following conditions holds,

(1) $D_{0}>0, z_{1}^{*}>0$ and $h\left(z_{1}^{*}\right)<0$;

(2) $D_{0}=0, z_{2}^{*}>0$ and $h\left(z_{2}^{*}\right)<0$;

(3) $D_{0}<0, z_{3}^{*}>0$ and $h\left(z_{3}^{*}\right)<0$,

then the order of all roots with positive real parts of (2.19) has the same sum as that of all roots with positive real parts of (2.19) for all $\tau \in\left[0, \tau_{0}\right)$.

Let

$$
\lambda_{k}(\tau)=\alpha_{k}(\tau) \pm i \omega_{k}(\tau), \quad k=1,2,3,4
$$

be the root of (2.1) near $\tau=\tau_{j}^{(k)}$ satisfying

$$
\alpha_{k}\left(\tau_{j}^{(k)}\right)=0, \quad \omega_{k}\left(\tau_{j}^{(k)}\right)=\omega_{k}, \quad k=1,2,3,4, j=0,1,2, \ldots
$$


8 A BAM neural network with multiple delays

Lemma 2.4. Suppose that $z_{k}=\omega_{k}^{2}$ and $d h\left(z_{k}\right) / d z \neq 0$. Then the following transversality conditions hold:

$$
\operatorname{Re}\left[\left.\frac{d \lambda_{k}(\tau)}{d \tau}\right|_{\tau=\tau_{j}^{(k)}}\right] \neq 0
$$

and the sign of $\operatorname{Re}\left[d \lambda_{k}(\tau) /\left.d \tau\right|_{\tau=\tau_{j}^{(k)}}\right]$ is the same as that of $d h\left(z_{k}\right) / d z$.

Proof. Differentiating the two sides of (2.1) with respect to $\tau$ and noticing that $\lambda$ is the function of $\tau$, one can obtain

$$
\begin{aligned}
\left\{4 \lambda^{3}\right. & \left.+3 a_{3} \lambda^{2}+2 a_{2} \lambda+a_{1}+\left[\left(2 b_{2} \lambda+b_{1}\right)-\tau\left(b_{2} \lambda^{2}+b_{1} \lambda+b_{0}\right)\right] e^{-\lambda \tau}\right\} \frac{d \lambda}{d \tau} \\
& =\left(b_{2} \lambda^{2}+b_{1} \lambda+b_{0}\right) \lambda e^{-\lambda \tau} .
\end{aligned}
$$

Thus, we have

$$
\left(\frac{d \lambda}{d \tau}\right)^{-1}=\frac{\left(4 \lambda^{3}+3 a_{3} \lambda^{2}+2 a_{2} \lambda+a_{1}\right) e^{\lambda \tau}}{\left(b_{2} \lambda^{2}+b_{1} \lambda+b_{0}\right) \lambda}+\frac{2 b_{2} \lambda+b_{1}}{\left(b_{2} \lambda^{2}+b_{1} \lambda+b_{0}\right) \lambda}-\frac{\tau}{\lambda}
$$

Notice that $\lambda= \pm i \omega_{k}$ when $\tau=\tau_{j}^{(k)}(k=1,2,3,4, j=0,1,2, \ldots)$ and we only need to consider the case that $\lambda=i \omega_{k}$ because the case $\lambda=-i \omega_{k}$ can be obtained similarly. Accordingly, when $\tau=\tau_{j}^{(k)}(k=1,2,3,4, j=0,1,2, \ldots)$, we have

$$
\begin{aligned}
& {\left[\left(b_{2} \lambda^{2}+b_{1} \lambda+b_{0}\right) \lambda\right]_{\tau=\tau_{j}^{(k)}} }\left(-b_{2} \omega_{k}^{2}+i b_{1} \omega_{k}+b_{0}\right) i \omega_{k}=-b_{1} \omega_{k}^{2}+i\left(b_{0}-b_{2} \omega_{k}^{2}\right) \omega_{k}, \\
& {\left[2 b_{2} \lambda+b_{1}\right]_{\tau=\tau_{j}^{(k)}}=b_{1}+2 i b_{2} \omega_{k}, } \\
& {\left[\left(4 \lambda^{3}+3 a_{3} \lambda^{2}+2 a_{2} \lambda+a_{1}\right) e^{\lambda \tau}\right]_{\tau=\tau_{j}^{(k)}} } \\
&=\left(-4 i \omega_{k}^{3}-3 a_{3} \omega_{k}^{2}+2 i a_{2} \omega_{k}+a_{1}\right)\left(\cos \omega_{k} \tau_{j}^{(k)}+i \sin \omega_{k} \tau_{j}^{(k)}\right) \\
&=\left[\left(a_{1}-3 a_{3} \omega_{k}^{2}\right) \cos \omega_{k} \tau_{j}^{(k)}-2 \omega_{k}\left(a_{2}-2 \omega_{k}^{2}\right) \sin \omega_{k} \tau_{j}^{(k)}\right] \\
& \quad+i\left[2 \omega_{k}\left(a_{2}-2 \omega_{k}^{2}\right) \cos \omega_{k} \tau_{j}^{(k)}+\left(a_{1}-3 a_{3} \omega_{k}^{2}\right) \sin \omega_{k} \tau_{j}^{(k)}\right] .
\end{aligned}
$$

Let

$$
M=\left|-b_{1} \omega_{k}^{2}+i\left(b_{0}-b_{2} \omega_{k}^{2}\right) \omega_{k}\right|^{2}=b_{1}^{2} \omega_{k}^{4}+\left(b_{0}-b_{2} \omega_{k}^{2}\right)^{2} \omega_{k}^{2}
$$


Then it follows from (2.3) and (2.25) that

$$
\begin{aligned}
\operatorname{Re}\left[\frac{\left(4 \lambda^{3}+3 a_{3} \lambda^{2}+2 a_{2} \lambda+a_{1}\right) e^{\lambda \tau}}{\left(b_{2} \lambda^{2}+b_{1} \lambda+b_{0}\right) \lambda}\right]_{\tau=\tau_{j}^{(k)}} & \frac{1}{M}\left\{-b_{1} \omega_{k}^{2}\left[\left(a_{1}-3 a_{3} \omega_{k}^{2}\right) \cos \omega_{k} \tau_{j}^{(k)}-2 \omega_{k}\left(a_{2}-2 \omega_{k}^{2}\right) \sin \omega_{k} \tau_{j}^{(k)}\right]\right. \\
& \left.\quad+\left(b_{0}-b_{2} \omega_{k}^{2}\right) \omega_{k}\left[2 \omega_{k}\left(a_{2}-2 \omega_{k}^{2}\right) \cos \omega_{k} \tau_{j}^{(k)}+\left(a_{1}-3 a_{3} \omega_{k}^{2}\right) \sin \omega_{k} \tau_{j}^{(k)}\right]\right\} \\
= & \frac{1}{M}\left\{\left(a_{1}-3 a_{3} \omega_{k}^{2}\right) \omega_{k}\left[-\left(b_{2} \omega_{k}^{2}-b_{0}\right) \sin \omega_{k} \tau_{j}^{(k)}-b_{1} \omega_{k} \cos \omega_{k} \tau_{j}^{(k)}\right]\right. \\
& \left.\quad+2\left(a_{2}-2 \omega_{k}^{2}\right) \omega_{k}^{2}\left[-\left(b_{2} \omega_{k}^{2}-b_{0}\right) \cos \omega_{k} \tau_{j}^{(k)}+b_{1} \omega_{k} \sin \omega_{k} \tau_{j}^{(k)}\right]\right\} \\
= & \frac{1}{M}\left[\left(a_{1}-3 a_{3} \omega_{k}^{2}\right) \omega_{k}\left(-a_{3} \omega_{k}^{3}+a_{1} \omega_{k}\right)-2\left(a_{2}-2 \omega_{k}^{2}\right) \omega_{k}^{2}\left(\omega_{k}^{4}-a_{2} \omega_{k}^{2}+a_{0}\right)\right] \\
= & \frac{\omega_{k}^{2}}{M}\left\{4 \omega_{k}^{6}+3\left(a_{3}^{2}-2 a_{2}\right) \omega_{k}^{4}+2\left(2 a_{0}+a_{2}^{2}-2 a_{1} a_{3}\right) \omega_{k}^{2}+a_{1}^{2}-2 a_{0} a_{2}\right\}, \\
\operatorname{Re}\left[\frac{2 b_{2} \lambda+b_{1}}{\left(b_{2} \lambda^{2}+b_{1} \lambda+b_{0}\right) \lambda}\right]_{\tau=\tau_{j}^{(k)}} & \frac{1}{M}\left[-b_{1}^{2} \omega_{k}^{2}+2 b_{2} \omega_{k}\left(b_{0}-b_{2} \omega_{k}^{2}\right) \omega_{k}\right]=\frac{\omega_{k}^{2}}{M}\left(-2 b_{2}^{2} \omega_{k}^{2}+2 b_{0} b_{2}-b_{1}^{2}\right) .
\end{aligned}
$$

Therefore, from (2.5) and (2.27), we can get

$$
\begin{aligned}
& \operatorname{Re}\left[\left(\left.\frac{d \lambda_{k}(\tau)}{d \tau}\right|_{\tau=\tau_{j}^{(k)}}\right)^{-1}\right] \\
& =\operatorname{Re}\left\{\left[\frac{\left(4 \lambda^{3}+3 a_{3} \lambda^{2}+2 a_{2} \lambda+a_{1}\right) e^{\lambda \tau}}{\left(b_{2} \lambda^{2}+b_{1} \lambda+b_{0}\right) \lambda}\right]_{\tau=\tau_{j}^{(k)}}\right\}+\operatorname{Re}\left\{\left[\frac{\left(2 b_{2} \lambda+b_{1}\right)}{\left(b_{2} \lambda^{2}+b_{1} \lambda+b_{0}\right) \lambda}\right]_{\tau=\tau_{j}^{(k)}}\right\} \\
& =\frac{\omega_{k}^{2}}{M}\left\{4 \omega_{k}^{6}+3\left(a_{3}^{2}-2 a_{2}\right) \omega_{k}^{4}+2\left(2 a_{0}+a_{2}^{2}-2 a_{1} a_{3}-b_{2}^{2}\right) \omega_{k}^{2}+a_{1}^{2}-2 a_{0} a_{2}+2 b_{0} b_{2}-b_{1}^{2}\right\} \\
& =\frac{z_{k}}{M}\left(4 z_{k}^{3}+3 a z 2+2 b z_{k}+c\right)=\frac{z_{k}}{M} \frac{d h\left(z_{k}\right)}{d z} \neq 0 .
\end{aligned}
$$

Thus, $\operatorname{Re}\left[d \lambda_{k}(\tau) /\left.d \tau\right|_{\tau=\tau_{j}^{(k)}}\right] \neq 0$. In addition, since $z_{k}>0$, we conclude that $\operatorname{Re}\left[d \lambda_{k}(\tau) /\right.$ $\left.\left.d \tau\right|_{\tau=\tau_{j}^{(k)}}\right]$ and $d h\left(z_{k}\right) / d z$ have the same sign. This completes the proof. 


\section{The simplified delayed four-neuron BAM neural network}

In this section, we will apply the results obtained in Section 2 to study the stability and bifurcation of the simplified BAM neural network (1.1).

Throughout this section, we will assume that the activation functions $f_{k}(k=1,2,3,4)$ of BAM network model (1.1) satisfy the following conditions:

$\left(\mathrm{H}_{1}\right) f_{k} \in C^{1}(\mathbb{R}, \mathbb{R}), f_{k}(0)=0$.

Under the assumption $\left(\mathrm{H}_{1}\right)$, we know easily that $(0,0,0,0)$ is an equilibrium of system $(1.1)$ and let $u_{k}(t)(k=1,2,3,4)$ be defined respectively by $u_{1}(t)=x_{1}\left(t-\tau_{1}\right), u_{k}(t)=$ $x_{k}(t)(k=2,3,4)$ and $\tau=\tau_{1}+\tau_{2}$, then system (1.1) is equivalent to the following system

$$
\begin{aligned}
\dot{u}_{1}(t)=-\mu_{1} u_{1}(t)+ & c_{21} f_{1}\left(u_{2}(t-\tau)\right)+c_{31} f_{1}\left(u_{3}(t-\tau)\right)+c_{41} f_{1}\left(u_{4}(t-\tau)\right), \\
\dot{u}_{2}(t) & =-\mu_{2} u_{2}(t)+c_{12} f_{2}\left(u_{1}(t)\right), \\
& \dot{u}_{3}(t)=-\mu_{3} u_{3}(t)+c_{13} f_{3}\left(u_{1}(t)\right) \\
\dot{u}_{4}(t) & =-\mu_{4} u_{4}(t)+c_{14} f_{4}\left(u_{1}(t)\right) .
\end{aligned}
$$

Linearized system of (3.1) near the equilibrium $(0,0,0,0)$ gives the following linear system

$$
\begin{aligned}
\dot{u}_{1}(t)=-\mu_{1} u_{1}(t)+\alpha_{21} u_{2}(t-\tau)+\alpha_{31} u_{3}(t-\tau)+\alpha_{41} u_{4}(t-\tau), & \\
\dot{u}_{2}(t) & =-\mu_{2} u_{2}(t)+\alpha_{12} u_{1}(t), \\
\dot{u}_{3}(t) & =-\mu_{3} u_{3}(t)+\alpha_{13} u_{1}(t), \\
\dot{u}_{4}(t) & =-\mu_{4} u_{4}(t)+\alpha_{14} u_{1}(t)
\end{aligned}
$$

where $\alpha_{k 1}=c_{k 1} f_{1}^{\prime}(0)(k=2,3,4)$ and $\alpha_{1 l}=c_{1 l} f_{l}^{\prime}(0)(l=2,3,4)$. The corresponding characteristic equation of system (3.2) is given by

$$
\operatorname{det}\left[\begin{array}{cccc}
\lambda+\mu_{1} & -\alpha_{21} e^{-\lambda \tau} & -\alpha_{31} e^{-\lambda \tau} & -\alpha_{41} e^{-\lambda \tau} \\
-\alpha_{12} & \lambda+\mu_{2} & 0 & 0 \\
-\alpha_{13} & 0 & \lambda+\mu_{3} & 0 \\
-\alpha_{14} & 0 & 0 & \lambda+\mu_{4}
\end{array}\right]=0,
$$

that is

$$
\begin{aligned}
\lambda^{4}+\left(\mu_{1}\right. & \left.+\mu_{2}+\mu_{3}+\mu_{4}\right) \lambda^{3}+\left(\mu_{1} \mu_{2}+\mu_{1} \mu_{3}+\mu_{1} \mu_{4}+\mu_{2} \mu_{3}+\mu_{2} \mu_{4}+\mu_{3} \mu_{4}\right) \lambda^{2} \\
+ & \left(\mu_{1} \mu_{2} \mu_{3}+\mu_{1} \mu_{2} \mu_{4}+\mu_{1} \mu_{3} \mu_{4}+\mu_{2} \mu_{3} \mu_{4}\right) \lambda+\mu_{1} \mu_{2} \mu_{3} \mu_{4} \\
- & \left\{\left(\alpha_{12} \alpha_{21}+\alpha_{13} \alpha_{31}+\alpha_{14} \alpha_{41}\right) \lambda^{2}\right. \\
+ & {\left[\alpha_{12} \alpha_{21}\left(\mu_{3}+\mu_{4}\right)+\alpha_{13} \alpha_{31}\left(\mu_{2}+\mu_{4}\right)+\alpha_{14} \alpha_{41}\left(\mu_{2}+\mu_{3}\right)\right] \lambda } \\
& \left.+\alpha_{12} \alpha_{21} \mu_{3} \mu_{4}+\alpha_{13} \alpha_{31} \mu_{2} \mu_{4}+\alpha_{14} \alpha_{41} \mu_{2} \mu_{3}\right\} e^{-\lambda \tau}=0 .
\end{aligned}
$$


In the sequel, we apply the results obtained in Section 2 to investigate (3.4), and obtain the sufficient conditions ensuring the stability of the equilibrium $(0,0,0,0)$ and Hopf bifurcation of system (1.1). From (2.1) and (3.4), one can see easily that

$$
\begin{gathered}
a_{3}=\mu_{1}+\mu_{2}+\mu_{3}+\mu_{4}>0 \\
a_{2}=\mu_{1} \mu_{2}+\mu_{1} \mu_{3}+\mu_{1} \mu_{4}+\mu_{2} \mu_{3}+\mu_{2} \mu_{4}+\mu_{3} \mu_{4}>0 \\
a_{1}=\mu_{1} \mu_{2} \mu_{3}+\mu_{1} \mu_{2} \mu_{4}+\mu_{1} \mu_{3} \mu_{4}+\mu_{2} \mu_{3} \mu_{4}>0 \\
a_{0}=\mu_{1} \mu_{2} \mu_{3} \mu_{4}>0 \\
b_{2}=-\left(\alpha_{12} \alpha_{21}+\alpha_{13} \alpha_{31}+\alpha_{14} \alpha_{41}\right), \\
b_{1}=-\left[\alpha_{12} \alpha_{21}\left(\mu_{3}+\mu_{4}\right)+\alpha_{13} \alpha_{31}\left(\mu_{2}+\mu_{4}\right)+\alpha_{14} \alpha_{41}\left(\mu_{2}+\mu_{3}\right)\right], \\
b_{0}=-\left(\alpha_{12} \alpha_{21} \mu_{3} \mu_{4}+\alpha_{13} \alpha_{31} \mu_{2} \mu_{4}+\alpha_{14} \alpha_{41} \mu_{2} \mu_{3}\right),
\end{gathered}
$$

which, together with (2.5), means that

$$
\begin{aligned}
a= & a_{3}^{2}-2 a_{2}=\mu_{1}^{2}+\mu_{2}^{2}+\mu_{3}^{2}+\mu_{4}^{2}>0, \\
b= & 2 a_{0}+a_{2}^{2}-2 a_{1} a_{3}-b_{2}^{2}=\mu_{1}^{2} \mu_{2}^{2}+\mu_{1}^{2} \mu_{3}^{2}+\mu_{1}^{2} \mu_{4}^{2}+\mu_{2}^{2} \mu_{3}^{2}+\mu_{2}^{2} \mu_{4}^{2}+\mu_{3}^{2} \mu_{4}^{2} \\
& -\left(\alpha_{12} \alpha_{21}+\alpha_{13} \alpha_{31}+\alpha_{14} \alpha_{41}\right)^{2}, \\
c= & a_{1}^{2}-2 a_{0} a_{2}+2 b_{0} b_{2}-b_{1}^{2}=\mu_{1}^{2} \mu_{2}^{2} \mu_{3}^{2}+\mu_{1}^{2} \mu_{2}^{2} \mu_{4}^{2}+\mu_{1}^{2} \mu_{3}^{2} \mu_{4}^{2}+\mu_{2}^{2} \mu_{3}^{2} \mu_{4}^{2} \\
& +2\left(\alpha_{12} \alpha_{21}+\alpha_{13} \alpha_{31}+\alpha_{14} \alpha_{41}\right)\left(\alpha_{12} \alpha_{21} \mu_{3} \mu_{4}+\alpha_{13} \alpha_{31} \mu_{2} \mu_{4}+\alpha_{14} \alpha_{41} \mu_{2} \mu_{3}\right) \\
& -\left[\alpha_{12} \alpha_{21}\left(\mu_{3}+\mu_{4}\right)+\alpha_{13} \alpha_{31}\left(\mu_{2}+\mu_{4}\right)+\alpha_{14} \alpha_{41}\left(\mu_{2}+\mu_{3}\right)\right]^{2}, \\
d= & a_{0}^{2}-b_{0}^{2}=\mu_{1}^{2} \mu_{2}^{2} \mu_{3}^{2} \mu_{4}^{2}-\left(\alpha_{12} \alpha_{21} \mu_{3} \mu_{4}+\alpha_{13} \alpha_{31} \mu_{2} \mu_{4}+\alpha_{14} \alpha_{41} \mu_{2} \mu_{3}\right)^{2} .
\end{aligned}
$$

Thus, we can calculate (2.7) and (2.9). Furthermore, from the sign of $D_{0}$, we can also know $z_{1}^{*}\left(D_{0}>0\right), z_{2}^{*}\left(D_{0}=0\right)$ or $z_{3}^{*}\left(D_{0}<0\right)$. In addition, in view of Routh-Hurwitz criteria, we can easily know that all roots of (3.4) with $\tau=0$ have negative real parts if the following condition holds:

$\left(\mathrm{H}_{2}\right) a_{0}+b_{0}>0, a_{1}+b_{1}>0, a_{3}>0$ and $a_{3}\left[\left(a_{1}+b_{1}\right)\left(a_{2}+b_{2}\right)-a_{3}\left(a_{0}+b_{0}\right)\right]>\left(a_{1}+\right.$ $\left.b_{1}\right)^{2}$.

By Lemmas 2.3 and 2.4, we have the following result regarding on stability and bifurcation of system (1.1).

Theorem 3.1. Let $a_{k}, b_{l}(k=0,1,2,3 ; l=0,1,2), a, b, c, d$, and $\tau_{j}^{(k)}$ be defined, respectively, by (3.5), (3.6), and (2.16). Suppose that $\left(H_{1}\right)$ and $\left(H_{2}\right)$ hold. Then, for system (3.1), the following statements are true.

(i) All roots of (3.4) have negative real parts and the zero solution of system (3.1) is absolutely stable if $d \geq 0$ and one of the following conditions is satisfied:

(1) $D_{0}>0$ and $z_{1}^{*}<0$;

(2) $D_{0}=0$ and $z_{2}^{*}<0$;

(3) $D_{0}<0$ and $z_{3}^{*}<0$. 
12 A BAM neural network with multiple delays

(ii) All roots of (3.4) have negative real parts and hence the zero solution of system (3.1) is asymptotically stable for $\tau \in\left[0, \tau_{0}\right)$ if $d<0$ or $d \geq 0$ and one of the following conditions holds:

(1) $D_{0}>0, z_{1}^{*}>0$ and $h\left(z_{1}^{*}\right)<0$;

(2) $D_{0}=0, z_{2}^{*}>0$ and $h\left(z_{2}^{*}\right)<0$;

(3) $D_{0}<0, z_{3}^{*}>0$ and $h\left(z_{3}^{*}\right)<0$.

(iii) $h(z)$ has at least a positive root $z_{k}$ and $\tau \in\left\{\tau_{j}^{(k)}\right\}, k=1,2,3,4 ; j=0,1,2, \ldots$, are Hopf bifurcation values for system (3.1) if the conditions as stated in (ii) are satisfied and $h^{\prime}\left(z_{k}\right) \neq 0$.

\section{Direction and stability of the Hopf local bifurcation}

In the above section, we have already obtained some sufficient conditions ensuring system (3.1) undergoes a Hopf bifurcation at the equilibrium $(0,0,0,0)$ when $\tau$ takes some certain critical values. In this section, we suppose that a Hopf bifurcation for system (3.1) will occur at the zero equilibrium when $\tau=\tau_{j}(j=0,1,2, \ldots)$, that is, a family of periodic solutions bifurcate from the zero equilibrium and will establish the explicit formulae determining the direction, stability, and period of these periodic solutions bifurcating from the zero equilibrium at these critical values $\tau_{j}(j=0,1,2, \ldots)$ of $\tau$ by using the normal theory and the center manifold argument developed by Hassard et al. [10]. Without loss of generality, we denote any one of these critical values $\tau=\tau_{j}(j=0,1,2, \ldots)$ by $\tilde{\tau}$, at which (3.4) has a pair of purely imaginary roots $\pm i \omega$ and system (3.1) undergoes a Hopf bifurcation from the zero equilibrium.

Throughout this section, we make also the following for the conditions in $\left(\mathrm{H}_{1}\right)$ :

$\left(\mathrm{H}_{3}\right) f_{k} \in C^{3}(\mathbb{R}, \mathbb{R}), k=1,2,3,4$.

For the sake of simplicity, we denote $\tau$ as $\tau=\tilde{\tau}+\mu, \mu \in \mathbb{R}$. Then $\mu=0$ is the Hopf bifurcation value of system (3.1). Let $y_{k}(t)=u_{k}(\tau t)(k=1,2,3,4)$. Then system (3.1) can be rewritten an functional differential equation in $C\left([-1,0], \mathbb{R}^{4}\right)$ as

$$
\begin{aligned}
\dot{y}_{1}(t)=\tau\left[-\mu_{1} y_{1}(t)+c_{21}\right. & \left.f_{1}\left(y_{2}(t-1)\right)+c_{31} f_{1}\left(y_{3}(t-1)\right)+c_{41} f_{1}\left(y_{4}(t-1)\right)\right], \\
\dot{y}_{2}(t) & =\tau\left[-\mu_{2} y_{2}(t)+c_{12} f_{2}\left(y_{1}(t)\right)\right], \\
\dot{y}_{3}(t) & =\tau\left[-\mu_{3} y_{3}(t)+c_{13} f_{3}\left(y_{1}(t)\right)\right], \\
\dot{y}_{4}(t) & =\tau\left[-\mu_{4} y_{4}(t)+c_{14} f_{4}\left(y_{1}(t)\right)\right] .
\end{aligned}
$$

Let $y=\left(y_{1}, y_{2}, y_{3}, y_{4}\right)^{T}$ and substitute the Taylor expansion of $f_{k}(k=1,2,3,4)$ at the zero point into system (4.1), one can get the linearization of system (4.1) at the zero equilibrium $y=0$ as

$$
\begin{gathered}
\dot{y}_{1}(t)=(\tilde{\tau}+\mu)\left[-\mu_{1} y_{1}(t)+\alpha_{21} y_{2}(t-1)+\alpha_{31} y_{3}(t-1)+\alpha_{41} y_{4}(t-1)\right], \\
\dot{y}_{2}(t)=(\tilde{\tau}+\mu)\left[-\mu_{2} y_{2}(t)+\alpha_{12} y_{1}(t)\right], \\
\dot{y}_{3}(t)=(\tilde{\tau}+\mu)\left[-\mu_{3} y_{3}(t)+\alpha_{13} y_{1}(t)\right], \\
\dot{y}_{4}(t)=(\tilde{\tau}+\mu)\left[-\mu_{4} y_{4}(t)+\alpha_{14} y_{1}(t)\right] .
\end{gathered}
$$


Rewrite (4.2) as the following matrix vector form:

$$
\dot{y}(t)=(\tilde{\tau}+\mu)\left[B_{0} y(t)+B_{1} y(t-1)\right]=L_{\mu}\left(y_{t}\right)
$$

where

$$
B_{0}=\left[\begin{array}{cccc}
-\mu_{1} & 0 & 0 & 0 \\
\alpha_{12} & -\mu_{2} & 0 & 0 \\
\alpha_{13} & 0 & -\mu_{3} & 0 \\
\alpha_{14} & 0 & 0 & -\mu_{4}
\end{array}\right], \quad B_{1}=\left[\begin{array}{cccc}
0 & \alpha_{21} & \alpha_{31} & \alpha_{41} \\
0 & 0 & 0 & 0 \\
0 & 0 & 0 & 0 \\
0 & 0 & 0 & 0
\end{array}\right]
$$

For any $\phi=\left(\phi_{1}, \phi_{2}, \phi_{3}, \phi_{4}\right)^{T} \in C\left([-1,0], \mathbb{R}^{4}\right)$,

$$
L_{\mu}(\phi)=(\tilde{\tau}+\mu)\left[B_{0} \phi(0)+B_{1} \phi(-1)\right]
$$

Then $L_{\mu}$ is a continuous linear function mapping $C\left([-1,0], \mathbb{R}^{4}\right)$ into $\mathbb{R}^{4}$. By the Rieze representation theorem, there exists a $4 \times 4$ matrix function $\eta(\theta, \mu),-1 \leq \theta \leq 0$, whose elements are of bounded variation such that

$$
L_{\mu}(\phi)=\int_{-1}^{0} d \eta(\theta, \mu) \phi(\theta) \quad \text { for } \phi \in C\left([-1,0], \mathbb{R}^{4}\right)
$$

In fact, we can choose

$$
\eta(\theta, \mu)= \begin{cases}(\tilde{\tau}+\mu) B_{0}, & \theta=0 \\ 0, & \theta \in(-1,0) \\ -(\tilde{\tau}+\mu) B_{1}, & \theta=-1\end{cases}
$$

Then (4.6) is satisfied.

For $\phi \in C^{1}\left([-1,0], \mathbb{R}^{4}\right)$, define the operator $A(\mu)$ as

$$
A(\mu) \phi(\theta)= \begin{cases}\frac{d \phi(\theta)}{d \theta}, & \theta \in[-1,0) \\ L_{\mu}(\phi) \stackrel{\text { def }}{=} \int_{-1}^{0} d \eta(\xi, \mu) \phi(\xi), & \theta=0\end{cases}
$$

For $\phi=\left(\phi_{1}, \phi_{2}, \phi_{3}, \phi_{4}\right)^{T} \in C\left([-1,0], \mathbb{R}^{4}\right)$, let

$$
f(\mu, \phi)=(\tilde{\tau}+\mu)\left(\begin{array}{c}
k_{1} \phi_{2}^{2}(-1)+k_{2} \phi_{2}^{3}(-1)+k_{3} \phi_{3}^{2}(-1)+k_{4} \phi_{3}^{3}(-1) \\
+k_{5} \phi_{4}^{2}(-1)+k_{6} \phi_{4}^{3}(-1)+\cdots \\
l_{1} \phi_{1}^{2}(0)+l_{2} \phi_{1}^{3}(0)+\cdots \\
m_{1} \phi_{1}^{2}(0)+m_{2} \phi_{1}^{3}(0)+\cdots \\
n_{1} \phi_{1}^{2}(0)+n_{2} \phi_{1}^{3}(0)+\cdots
\end{array}\right)
$$


14 A BAM neural network with multiple delays

where

$$
\begin{aligned}
k_{1}=\frac{c_{21} f_{1}^{\prime \prime}(0)}{2 !}, & k_{2}=\frac{c_{21} f_{1}^{\prime \prime \prime}(0)}{3 !}, & k_{3}=\frac{c_{31} f_{1}^{\prime \prime}(0)}{2 !}, & k_{4}=\frac{c_{31} f_{1}^{\prime \prime \prime}(0)}{3 !}, \\
k_{5}=\frac{c_{41} f_{1}^{\prime \prime}(0)}{2 !}, & k_{6}=\frac{c_{41} f_{1}^{\prime \prime \prime}(0)}{3 !}, & l_{1}=\frac{c_{12} f_{2}^{\prime \prime}(0)}{2 !}, & l_{2}=\frac{c_{12} f_{2}^{\prime \prime \prime}(0)}{3 !}, \\
m_{1}=\frac{c_{13} f_{3}^{\prime \prime}(0)}{2 !}, & m_{2}=\frac{c_{13} f_{3}^{\prime \prime \prime}(0)}{3 !}, & n_{1}=\frac{c_{14} f_{4}^{\prime \prime}(0)}{2 !}, & n_{2}=\frac{c_{14} f_{4}^{\prime \prime \prime}(0)}{3 !} .
\end{aligned}
$$

If we further define the operator $R(\mu)$ as

$$
R(\mu) \phi(\theta)= \begin{cases}0, & \theta \in[-1,0) \\ f(\mu, \phi), & \theta=0\end{cases}
$$

then system (4.1) is equivalent to

$$
\dot{y}_{t}=A(\mu) y_{t}+R(\mu) y_{t} .
$$

For $\psi \in C^{1}\left([0,1],\left(\mathbb{R}^{4}\right)^{*}\right)$, define

$$
A^{*} \psi(s)= \begin{cases}-\frac{d \psi(s)}{d s}, & s \in(0,1], \\ \int_{-1}^{0} \psi(-\xi) d \eta(\xi, 0), & s=0,\end{cases}
$$

and a bilinear inner product

$$
\langle\psi(s), \phi(\theta)\rangle=\bar{\psi}(0) \phi(0)-\int_{-1}^{0} \int_{\xi=0}^{\theta} \bar{\psi}(\xi-\theta) d \eta(\theta) \phi(\xi) d \xi,
$$

where $\eta(\theta)=\eta(\theta, 0)$. Then $A(0)$ and $A^{*}$ are adjoint operators.

From the discussion in Section 2, we know that $\pm i \omega \tilde{\tau}$ are eigenvalues of $A(0)$ and therefore they are also eigenvalues of $A^{*}$. It is not difficult to verify the vector

$$
q(\theta)=\left(1, \frac{\alpha_{12}}{\mu_{2}+i \omega}, \frac{\alpha_{13}}{\mu_{3}+i \omega}, \frac{\alpha_{14}}{\mu_{4}+i \omega}\right)^{T} e^{i \omega \tilde{\tau} \theta}, \quad \theta \in[-1,0]
$$

is the eigenvector of $A(0)$ corresponding to the eigenvalue $i \omega \tilde{\tau}$, and

$$
q^{*}(s)=Q\left(1, \frac{\alpha_{21} e^{i \omega \tilde{\tau}}}{\mu_{2}-i \omega}, \frac{\alpha_{31} e^{i \omega \tilde{\tau}}}{\mu_{3}-i \omega}, \frac{\alpha_{41} e^{i \omega \tilde{\tau}}}{\mu_{4}-i \omega}\right) e^{i \omega \tilde{\tau} s}, \quad s \in[0,1],
$$

is the eigenvector of $A^{*}$ corresponding to the eigenvalue $-i \omega \tilde{\tau}$. Moreover, $\left\langle q^{*}(s), q(\theta)\right\rangle=$ 1 , where

$Q=\left[e^{-i \omega \tilde{\tau}}+\frac{\alpha_{12} \alpha_{21}}{\left(\mu_{2}-i \omega\right)^{2}}+\frac{\alpha_{12} \alpha_{21} \tilde{\tau}}{\mu_{2}-i \omega}+\frac{\alpha_{13} \alpha_{31}}{\left(\mu_{3}-i \omega\right)^{2}}+\frac{\alpha_{13} \alpha_{31} \tilde{\tau}}{\mu_{3}-i \omega}+\frac{\alpha_{14} \alpha_{41}}{\left(\mu_{4}-i \omega\right)^{2}}+\frac{\alpha_{14} \alpha_{41} \tilde{\tau}}{\mu_{4}-i \omega}\right]^{-1} e^{-i \omega \tilde{\tau}}$. 
Using the same notations as in Hassard et al. [10], we first compute the coordinates to describe the center manifold $C_{0}$ at $\mu=0$. Let $y_{t}=\left(y_{t}^{(1)}, y_{t}^{(2)}, y_{t}^{(3)}, y_{t}^{(4)}\right)^{T}$ be the solution of system (4.1) when $\mu=0$. Define

$$
\begin{gathered}
z(t)=\left\langle q^{*}(s), y_{t}(\theta)\right\rangle, \\
W(t, \theta)=y_{t}(\theta)-2 \operatorname{Re}\{z(t) q(\theta)\} .
\end{gathered}
$$

On the center manifold $C_{0}$, we have

$$
W(t, \theta)=W(z(t), \bar{z}(t), \theta)
$$

where

$$
W(z, \bar{z}, \theta)=W_{20}(\theta) \frac{z^{2}}{2}+W_{11}(\theta) z \bar{z}+W_{02}(\theta) \frac{\bar{z}^{2}}{2}+W_{30}(\theta) \frac{z^{3}}{6}+\cdots,
$$

$z$ and $\bar{z}$ are local coordinates for center manifold $C_{0}$ in the direction of $q$ and $q^{*}$. Note that $W$ is real if $y_{t}$ is real. We only consider real solution.

For solution $y_{t} \in C_{0}$ of system (4.1), we from (4.12) get $\dot{y}_{t}=A(0) y_{t}+R(0) y_{t}$ since $\mu=0$. Therefore, from the definition of $R(\mu)$ and (4.19), we have

$$
\begin{aligned}
\dot{z}(t) & =\left\langle q^{*}(s), \dot{y}_{t}\right\rangle=\left\langle q^{*}(s), A(0) y_{t}+R(0) y_{t}\right\rangle \\
& =\left\langle q^{*}(s), A(0) y_{t}\right\rangle+\left\langle q^{*}(s), R(0) y_{t}\right\rangle \\
& =\left\langle-i \omega \tilde{\tau} q^{*}(s), y_{t}\right\rangle+\bar{q}^{*}(0) f\left(0, y_{t}(\theta)\right) \\
& \stackrel{\text { def }}{=} i \omega \tilde{\tau} z(t)+\bar{q}^{*}(0) f_{0}(z(t), \bar{z}(t)) .
\end{aligned}
$$

We rewrite this as

$$
\dot{z}(t)=i \omega \tilde{\tau} z(t)+g(z(t), \bar{z}(t)),
$$

where

$$
g(z, \bar{z})=g_{20} \frac{z^{2}}{2}+g_{11} z \bar{z}+g_{02} \frac{\bar{z}^{2}}{2}+g_{21} \frac{z^{2} \bar{z}}{2}+\cdots .
$$

For the sake of simplicity, let

$$
\begin{array}{ccc}
\alpha=\frac{\alpha_{12}}{\mu_{2}+i \omega}, & \beta=\frac{\alpha_{13}}{\mu_{3}+i \omega}, & \gamma=\frac{\alpha_{14}}{\mu_{4}+i \omega}, \\
\alpha^{*}=\frac{\alpha_{21} e^{i \omega \tilde{\tau}}}{\mu_{2}-i \omega}, & \beta^{*}=\frac{\alpha_{31} e^{i \omega \tilde{\tau}}}{\mu_{3}-i \omega}, & \gamma^{*}=\frac{\alpha_{41} e^{i \omega \tilde{\tau}}}{\mu_{4}-i \omega} .
\end{array}
$$

Then (4.19) leads to

$$
\begin{aligned}
y_{t}(\theta) & =W(t, \theta)+2 \operatorname{Re}\{z(t) q(\theta)\}=W(t, \theta)+z(t) q(\theta)+\bar{z}(t) \bar{q}(\theta) \\
& =W_{20}(\theta) \frac{z^{2}}{2}+W_{11}(\theta) z \bar{z}+W_{02}(\theta) \frac{\bar{z}^{2}}{2}+(1, \alpha, \beta, \gamma)^{T} e^{i \omega \tilde{\tau} \theta} z+(1, \bar{\alpha}, \bar{\beta}, \bar{\gamma})^{T} e^{-i \omega \tilde{\tau} \theta} \bar{z}+\cdots
\end{aligned}
$$


Thus, we can easily obtain

$$
\begin{aligned}
& y_{t}^{(1)}(0)=W_{20}^{(1)}(0) \frac{z^{2}}{2}+W_{11}^{(1)}(0) z \bar{z}+W_{02}^{(1)}(0) \frac{\bar{z}^{2}}{2}+z+\bar{z}+\cdots, \\
& y_{t}^{(2)}(-1)=W_{20}^{(2)}(-1) \frac{z^{2}}{2}+W_{11}^{(2)}(-1) z \bar{z}+W_{02}^{(2)}(-1) \frac{\bar{z}^{2}}{2}+\alpha z e^{-i \omega \tilde{\tau}}+\bar{\alpha} \bar{z} e^{i \omega \tilde{\tau}}+\cdots, \\
& y_{t}^{(3)}(-1)=W_{20}^{(3)}(-1) \frac{z^{2}}{2}+W_{11}^{(3)}(-1) z \bar{z}+W_{02}^{(3)}(-1) \frac{\bar{z}^{2}}{2}+\beta z e^{-i \omega \tilde{\tau}}+\bar{\beta} \bar{z} e^{i \omega \tilde{\tau}}+\cdots, \\
& y_{t}^{(4)}(-1)=W_{20}^{(4)}(-1) \frac{z^{2}}{2}+W_{11}^{(4)}(-1) z \bar{z}+W_{02}^{(4)}(-1) \frac{\bar{z}^{2}}{2}+\gamma z e^{-i \omega \tilde{\tau}}+\bar{\gamma} \bar{z} e^{i \omega \tau}+\cdots
\end{aligned}
$$

Therefore, it follows from (4.9) that

$$
f_{0}(z(t), \bar{z}(t)) \quad\left(\begin{array}{c}
2\left(k_{1} \alpha^{2}+k_{3} \beta^{2}+k_{5} \gamma^{2}\right) e^{-2 i \omega \tilde{\tau}} \frac{z^{2}}{2}+2\left(k_{1}|\alpha|^{2}+k_{3}|\beta|^{2}+k_{5}|\gamma|^{2}\right) z \bar{z} \\
+2\left(k_{1} \bar{\alpha}^{2}+k_{3} \bar{\beta}^{2}+k_{5} \bar{\gamma}^{2}\right) e^{2 i \omega \tau} \frac{\bar{z}^{2}}{2} \\
+2\left[\left(k_{1} W_{20}^{(2)}(-1) \bar{\alpha}+k_{3} W_{20}^{(3)}(-1) \bar{\beta}+k_{5} W_{20}^{(4)}(-1) \bar{\gamma}\right) e^{i \omega \tilde{\tau}}\right. \\
+2\left(k_{1} W_{11}^{(2)}(-1) \alpha+k_{3} W_{11}^{(3)}(-1) \beta+k_{5} W_{11}^{(4)}(-1) \gamma\right) e^{-i \omega \tilde{\tau}} \\
\left.+3\left(k_{2} \alpha^{2} \bar{\alpha}+k_{4} \beta^{2} \bar{\beta}+k_{6} \gamma^{2} \bar{\gamma}\right) e^{-i \omega \tau}\right] \frac{z^{2} \bar{z}}{2}+\cdots \\
2\left\{l_{1} \frac{z^{2}}{2}+l_{1} z \bar{z}+l_{1} \frac{\bar{z}^{2}}{2}+\left[l_{1}\left(W_{20}^{(1)}(0)+2 W_{11}^{(1)}(0)\right)+3 l_{2}\right] \frac{z^{2} \bar{z}}{2}\right\}+\cdots \\
2\left\{\begin{array}{c}
\left.z_{1}^{2} \frac{z^{2}}{2}+m_{1} z \bar{z}+m_{1} \frac{\bar{z}^{2}}{2}+\left[m_{1}\left(W_{20}^{(1)}(0)+2 W_{11}^{(1)}(0)\right)+3 m_{2}\right] \frac{z^{2} \bar{z}}{2}\right\}+\cdots \\
2\left\{n_{1} \frac{z^{2}}{2}+n_{1} z \bar{z}+n_{1} \frac{\bar{z}^{2}}{2}+\left[n_{1}\left(W_{20}^{(1)}(0)+2 W_{11}^{(1)}(0)\right)+3 n_{2}\right] \frac{z^{2} \bar{z}}{2}\right\}+\cdots
\end{array}\right.
\end{array}\right) .
$$

Thus one can obtain

$$
\begin{aligned}
g(z, \bar{z})= & g_{20} \frac{z^{2}}{2}+g_{11} z \bar{z}+g_{02} \frac{\bar{z}^{2}}{2}+g_{21} \frac{z^{2} \bar{z}}{2}+\cdots \\
= & \bar{q}^{*}(0) f_{0}(z(t), \bar{z}(t)) \\
= & 2 \bar{Q} \tilde{\tau}\left\{\left(k_{1} \alpha^{2}+k_{3} \beta^{2}+k_{5} \gamma^{2}\right) e^{-2 i \omega \tilde{\tau}}+l_{1} \overline{\alpha^{*}}+m_{1} \overline{\beta^{*}}+n_{1} \overline{\gamma^{*}}\right\} \frac{z^{2}}{2} \\
& +2 \bar{Q} \tilde{\tau}\left\{\left(k_{1}|\alpha|^{2}+k_{3}|\beta|^{2}+k_{5}|\gamma|^{2}\right)+l_{1} \overline{\alpha^{*}}+m_{1} \overline{\beta^{*}}+n_{1} \overline{\gamma^{*}}\right\} z \bar{z} \\
& +2 \bar{Q} \tilde{\tau}\left\{\left(k_{1} \bar{\alpha}^{2}+k_{3} \bar{\beta}^{2}+k_{5} \bar{\gamma}^{2}\right) e^{2 i \omega \tilde{\tau}}+l_{1} \overline{\alpha^{*}}+m_{1} \overline{\beta^{*}}+n_{1} \overline{\gamma^{*}}\right\} \frac{\bar{z}^{2}}{2}
\end{aligned}
$$




$$
\begin{aligned}
+2 \bar{Q} \tilde{\tau}\{ & \left(k_{1} W_{20}^{(2)}(-1) \bar{\alpha}+k_{3} W_{20}^{(3)}(-1) \bar{\beta}+k_{5} W_{20}^{(4)}(-1) \bar{\gamma}\right) e^{i \omega \tilde{\tau}} \\
& +2\left(k_{1} W_{11}^{(2)}(-1) \alpha+k_{3} W_{11}^{(3)}(-1) \beta+k_{5} W_{11}^{(4)}(-1) \gamma\right) e^{-i \omega \tilde{\tau}} \\
& +3\left(k_{2} \alpha^{2} \bar{\alpha}+k_{4} \beta^{2} \bar{\beta}+k_{6} \gamma^{2} \bar{\gamma}\right) e^{-i \omega \tilde{\tau}}+\overline{\alpha^{*}}\left[l_{1}\left(W_{20}^{(1)}(0)+2 W_{11}^{(1)}(0)\right)+3 l_{2}\right] \\
& +\overline{\beta^{*}}\left[m_{1}\left(W_{20}^{(1)}(0)+2 W_{11}^{(1)}(0)\right)+3 m_{2}\right] \\
& \left.+\overline{\gamma^{*}}\left[n_{1}\left(W_{20}^{(1)}(0)+2 W_{11}^{(1)}(0)\right)+3 n_{2}\right]\right\} \times \frac{z^{2} \bar{z}}{2}+\cdots
\end{aligned}
$$

Comparing the coefficients of two sides of (4.29), we have

$$
\begin{aligned}
g_{20}=2 \bar{Q} \tilde{\tau}[ & \left.\left(k_{1} \alpha^{2}+k_{3} \beta^{2}+k_{5} \gamma^{2}\right) e^{-2 i \omega \tilde{\tau}}+l_{1} \overline{\alpha^{*}}+m_{1} \overline{\beta^{*}}+n_{1} \overline{\gamma^{*}}\right], \\
g_{11}=2 \bar{Q} \tilde{\tau}[ & \left.\left(k_{1}|\alpha|^{2}+k_{3}|\beta|^{2}+k_{5}|\gamma|^{2}\right)+l_{1} \overline{\alpha^{*}}+m_{1} \overline{\beta^{*}}+n_{1} \overline{\gamma^{*}}\right], \\
g_{02}=2 \bar{Q} \tilde{\tau}[ & \left.\left(k_{1} \bar{\alpha}^{2}+k_{3} \bar{\beta}^{2}+k_{5} \bar{\gamma}^{2}\right) e^{2 i \omega \tilde{\tau}}+l_{1} \overline{\alpha^{*}}+m_{1} \overline{\beta^{*}}+n_{1} \overline{\gamma^{*}}\right], \\
g_{21}=2 \bar{Q} \tilde{\tau}\{ & \left(k_{1} W_{20}^{(2)}(-1) \bar{\alpha}+k_{3} W_{20}^{(3)}(-1) \bar{\beta}+k_{5} W_{20}^{(4)}(-1) \bar{\gamma}\right) e^{i \omega \tilde{\tau}} \\
& +2\left(k_{1} W_{11}^{(2)}(-1) \alpha+k_{3} W_{11}^{(3)}(-1) \beta+k_{5} W_{11}^{(4)}(-1) \gamma\right) e^{-i \omega \tilde{\tau}} \\
& +3\left(k_{2} \alpha^{2} \bar{\alpha}+k_{4} \beta^{2} \bar{\beta}+k_{6} \gamma^{2} \bar{\gamma}\right) e^{-i \omega \tilde{\tau}}+\overline{\alpha^{*}}\left[l_{1}\left(W_{20}^{(1)}(0)+2 W_{11}^{(1)}(0)\right)+3 l_{2}\right] \\
& \left.+\overline{\beta^{*}}\left[m_{1}\left(W_{20}^{(1)}(0)+2 W_{11}^{(1)}(0)\right)+3 m_{2}\right]+\overline{\gamma^{*}}\left[n_{1}\left(W_{20}^{(1)}(0)+2 W_{11}^{(1)}(0)\right)+3 n_{2}\right]\right\} .
\end{aligned}
$$

Since $W_{20}(\theta)$ and $W_{11}(\theta)$ for $\theta \in[-1,0]$ appear in $g_{21}$, we still need to compute them. From (4.12), (4.19), and (4.22), we have

$$
\begin{aligned}
\dot{W}= & \dot{y}_{t}-2 \operatorname{Re}\{\dot{z}(t) q(\theta)\} \\
& = \begin{cases}A(0) W(t, \theta)-2 \operatorname{Re}\left\{\bar{q}^{*}(0) f_{0}(z(t), \bar{z}(t)) q(\theta)\right\}, & \theta \in[-1,0), \\
A(0) W(t, \theta)-2 \operatorname{Re}\left\{\bar{q}^{*}(0) f_{0}(z(t), \bar{z}(t)) q(0)\right\}+f_{0}(z(t), \bar{z}(t)), & \theta=0,\end{cases} \\
& \stackrel{\text { def }}{=} A(0) W(t, \theta)+H(z(t), \bar{z}(t), \theta),
\end{aligned}
$$

where

$$
H(z, \bar{z}, \theta)=H_{20}(\theta) \frac{z^{2}}{2}+H_{11}(\theta) z \bar{z}+H_{02}(\theta) \frac{\bar{z}^{2}}{2}+\cdots
$$


18 A BAM neural network with multiple delays

From (4.31) and (4.32), we have

$$
A(0) W(t, \theta)-\dot{W}=-H(z, \bar{z}, \theta)=-H_{20}(\theta) \frac{z^{2}}{2}-H_{11}(\theta) z \bar{z}-H_{02}(\theta) \frac{\bar{z}^{2}}{2}-\cdots
$$

In view of (4.21), one can obtain

$$
\begin{gathered}
A(0) W(t, \theta)=A(0) W_{20}(\theta) \frac{z^{2}}{2}+A(0) W_{11}(\theta) z \bar{z}+\cdots, \\
\dot{W}=W_{z} \dot{z}+W_{\bar{z}} \dot{\bar{z}}=2 i \omega \tilde{\tau} W_{20}(\theta) \frac{z^{2}}{2}+\cdots
\end{gathered}
$$

It follows from (4.34) that

$$
A(0) W(t, \theta)-\dot{W}=[A(0)-2 i \omega \tilde{\tau} I] W_{20}(\theta) \frac{z^{2}}{2}+A(0) W_{11}(\theta) z \bar{z}+\cdots
$$

Thus, (4.33) and (4.35) imply that

$$
\begin{aligned}
& {[A(0)-2 i \omega \tilde{\tau} I] W_{20}(\theta) \frac{z^{2}}{2}+A(0) W_{11}(\theta) z \bar{z}+\cdots} \\
& \quad=-H_{20}(\theta) \frac{z^{2}}{2}-H_{11}(\theta) z \bar{z}-H_{02}(\theta) \frac{\bar{z}^{2}}{2}-\cdots
\end{aligned}
$$

Comparing the coefficients of two sides of (4.36), we have

$$
[A(0)-2 i \omega \tilde{\tau} I] W_{20}(\theta)=-H_{20}(\theta), \quad A(0) W_{11}(\theta)=-H_{11}(\theta), \ldots
$$

From (4.31), we know that for $\theta \in[-1,0)$,

$$
\begin{aligned}
H(z, \bar{z}, \theta) & =-2 \operatorname{Re}\left\{\bar{q}^{*}(0) f_{0}(z(t), \bar{z}(t)) q(\theta)\right\} \\
& =-\bar{q}^{*}(0) f_{0}(z(t), \bar{z}(t)) q(\theta)-q^{*}(0) \overline{f_{0}}(z(t), \bar{z}(t)) \bar{q}(\theta) \\
& =-g(z, \bar{z}) q(\theta)-\bar{g}(z, \bar{z}) \bar{q}(\theta) \\
& =-\left(g_{20} q(\theta)+\bar{g}_{02} \bar{q}(\theta)\right) \frac{z^{2}}{2}-\left(g_{11} q(\theta)+\bar{g}_{11} \bar{q}(\theta)\right) z \bar{z}+\cdots
\end{aligned}
$$

Comparing the coefficients with (4.32) gives that

$$
\begin{aligned}
& H_{20}(\theta)=-\left(g_{20} q(\theta)+\bar{g}_{02} \bar{q}(\theta)\right), \\
& H_{11}(\theta)=-\left(g_{11} q(\theta)+\bar{g}_{11} \bar{q}(\theta)\right) .
\end{aligned}
$$

From (4.37), (4.39) and the definition of $A(0)$, we have

$$
\dot{W}_{20}(\theta)=2 i \omega \tilde{\tau} W_{20}(\theta)+g_{20} q(\theta)+\bar{g}_{02} \bar{q}(\theta) .
$$


Note that $q(\theta)=q(0) e^{i \omega \tilde{\tau} \theta}$, hence

$$
W_{20}(\theta)=\frac{i g_{20}}{\omega \tilde{\tau}} q(\theta)+\frac{i \bar{g}_{02}}{3 \omega \tilde{\tau}} \bar{q}(\theta)+E_{1} e^{2 i \omega \tilde{\tau} \theta}
$$

Similarly, from (4.37), (4.40), and the definition of $A(0)$, we have

$$
\begin{gathered}
\dot{W}_{11}(\theta)=g_{11} q(\theta)+\bar{g}_{11} \bar{q}(\theta), \\
W_{11}(\theta)=-\frac{i g_{11}}{\omega \tilde{\tau}} q(\theta)+\frac{i \bar{g}_{11}}{\omega \tilde{\tau}} \bar{q}(\theta)+E_{2} .
\end{gathered}
$$

In what follows, we will seek appropriate $E_{1}$ and $E_{2}$ in (4.42) and (4.43), respectively. It follows from the definition of $A(0)$ and (4.37) that

$$
\begin{gathered}
\int_{-1}^{0} d \eta(\theta) W_{20}(\theta)=2 i \omega \tilde{\tau} W_{20}(0)-H_{20}(0), \\
\int_{-1}^{0} d \eta(\theta) W_{11}(\theta)=-H_{11}(0) .
\end{gathered}
$$

Note that $q(\theta)$ is the eigenvector of $A(0)$ and from (4.42) and the definition of $A(0)$, we know that

$$
\begin{gathered}
\int_{-1}^{0} d \eta(\theta) W_{20}(\theta)=-g_{20} q(0)+\frac{\bar{g}_{02}}{3} \bar{q}(0)+\int_{-1}^{0} d \eta(\theta) e^{2 i \omega \tilde{\tau} \theta} E_{1} \\
2 i \omega \tilde{\tau} W_{20}(0)=-2 g_{20} q(0)-\frac{2 \bar{g}_{02}}{3} \bar{q}(0)+2 i \omega \tilde{\tau} E_{1} .
\end{gathered}
$$

Thus, (4.44) becomes

$$
-g_{20} q(0)-\bar{g}_{02} \bar{q}(0)+\left(2 i \omega \tilde{\tau} I-\int_{-1}^{0} d \eta(\theta) e^{2 i \omega \tilde{\tau} \theta}\right) E_{1}=H_{20}(0) .
$$

Similarly, from (4.43), we have

$$
\int_{-1}^{0} d \eta(\theta) W_{11}(\theta)=g_{11} q(0)+\bar{g}_{11} \bar{q}(0)+\int_{-1}^{0} d \eta(\theta) E_{2}
$$

Hence (4.45) becomes

$$
-\left(g_{11} q(0)+\bar{g}_{11} \bar{q}(0)\right)-\int_{-1}^{0} d \eta(\theta) E_{2}=H_{11}(0) .
$$

From (4.31) and (4.32), we know that

$$
\begin{aligned}
H(z, \bar{z}, 0) & =H_{20}(0) \frac{z^{2}}{2}+H_{11}(0) z \bar{z}+H_{02}(0) \frac{\bar{z}^{2}}{2}+\cdots \\
& =-2 \operatorname{Re}\left\{\bar{q}^{*}(0) f_{0}(z(t), \bar{z}(t)) q(0)\right\}+f_{0}(z(t), \bar{z}(t)) \\
& =-\left(g_{20} q(0)+\bar{g}_{02} \bar{q}(0)\right) \frac{z^{2}}{2}-\left(g_{11} q(0)+\bar{g}_{11} \bar{q}(0)\right) z \bar{z}+f_{0}(z(t), \bar{z}(t))+\cdots
\end{aligned}
$$


It follows together with (4.28) that

$$
\begin{aligned}
& H_{20}(0)=-\left(g_{20} q(0)+\bar{g}_{02} \bar{q}(0)\right)+2 \tilde{\tau} h_{20}, \\
& H_{11}(0)=-\left(g_{11} q(0)+\bar{g}_{11} \bar{q}(0)\right)+2 \tilde{\tau} h_{11},
\end{aligned}
$$

where

$$
\begin{gathered}
h_{20}=\left(\left(k_{1} \alpha^{2}+k_{3} \beta^{2}+k_{5} \gamma^{2}\right) e^{-2 i \omega \tilde{\tau}}, l_{1}, m_{1}, n_{1}\right)^{T}, \\
h_{11}=\left(k_{1}|\alpha|^{2}+k_{3}|\beta|^{2}+k_{5}|\gamma|^{2}, l_{1}, m_{1}, n_{1}\right)^{T} .
\end{gathered}
$$

Substituting (4.51) into (4.47), we obtain

$$
\left(2 i \omega \tilde{\tau} I-\int_{-1}^{0} d \eta(\theta) e^{2 i \omega \tilde{\tau} \theta}\right) E_{1}=2 \tilde{\tau} h_{20},
$$

that is,

$$
\left[\begin{array}{cccc}
2 i \omega+\mu_{1} & -\alpha_{21} e^{-2 i \omega \tilde{\tau}} & -\alpha_{31} e^{-2 i \omega \tilde{\tau}} & -\alpha_{41} e^{-2 i \omega \tilde{\tau}} \\
-\alpha_{12} & 2 i \omega+\mu_{2} & 0 & 0 \\
-\alpha_{13} & 0 & 2 i \omega+\mu_{3} & 0 \\
-\alpha_{14} & 0 & 0 & 2 i \omega+\mu_{4}
\end{array}\right] E_{1}=2 h_{20}
$$

Solving this equation, one can obtain $E_{1}$.

Similarly, substituting (4.52) into (4.48), we get

$$
\int_{-1}^{0} d \eta(\theta) E_{2}=-2 \tilde{\tau} h_{11},
$$

which means that

$$
\left[\begin{array}{cccc}
\mu_{1} & -\alpha_{21} & -\alpha_{31} & -\alpha_{41} \\
-\alpha_{12} & \mu_{2} & 0 & 0 \\
-\alpha_{13} & 0 & \mu_{3} & 0 \\
-\alpha_{14} & 0 & 0 & \mu_{4}
\end{array}\right] E_{2}=2 h_{11}
$$

From this equation, we can get $E_{2}$.

Consequently, $g_{21}$ can be expressed explicitly. Thus, we can compute the following values:

$$
\begin{gathered}
c_{1}(0)=\frac{i}{2 \omega \tilde{\tau}}\left(g_{11} g_{20}-2\left|g_{11}\right|^{2}-\frac{\left|g_{02}\right|^{2}}{3}\right)+\frac{g_{21}}{2}, \\
\varrho_{2}=-\frac{\operatorname{Re}\left(c_{1}(0)\right)}{\operatorname{Re}\left(\lambda_{k}^{\prime}\left(\tau_{j}\right)\right)}, \quad \varsigma_{2}=2 \operatorname{Re}\left(c_{1}(0)\right), \\
T_{2}=-\frac{\operatorname{Im}\left(c_{1}(0)\right)+\sigma_{2} \operatorname{Im}\left(\lambda_{k}^{\prime}\left(\tau_{j}\right)\right)}{\omega}, \quad j=0,1,2, \ldots,
\end{gathered}
$$

which determine the properties of bifurcating periodic solutions at the critical value $\tau_{j}$, that is, $\varrho_{2}$ determines the directions of the Hopf bifurcation: if $\varrho_{2}>0\left(\varrho_{2}<0\right)$, then the 
Hopf bifurcation is supercritical (subcritical) and the bifurcating periodic solutions exist for $\tau>\tau_{j}\left(\tau<\tau_{j}\right)$; $\varsigma_{2}$ determines the stability of the bifurcating periodic solutions: the bifurcating periodic solutions on the center manifold are stable (unstable) if $\varsigma_{2}<0\left(\varsigma_{2}>0\right)$; and $T_{2}$ determines the period of the bifurcating periodic solutions: the period increases (decreases) if $T_{2}>0\left(T_{2}<0\right)$.

\section{Applications and numerical simulations}

In this section, we will apply the results obtained in Sections 3 and 4 to investigate a special four-neuron BAM neural network with two delays and give numerical simulations supporting our theoretical analysis.

5.1. Application to a special BAM neural network. Consider the four-neuron BAM neural network with two delays described by the following functional differential equations:

$$
\begin{aligned}
\dot{x}_{1}(t)=-\mu x_{1}(t)+c_{21} f\left(x_{2}\left(t-\tau_{2}\right)\right)+c_{31} f\left(x_{3}\left(t-\tau_{2}\right)\right)+c_{41} f\left(x_{4}\left(t-\tau_{2}\right)\right), \\
\dot{x}_{2}(t)=-\mu x_{2}(t)+c_{12} f\left(x_{1}\left(t-\tau_{1}\right)\right), \\
\dot{x}_{3}(t)=-\mu x_{3}(t)+c_{13} f\left(x_{1}\left(t-\tau_{1}\right)\right), \\
\dot{x}_{4}(t)=-\mu x_{4}(t)+c_{14} f\left(x_{1}\left(t-\tau_{1}\right)\right),
\end{aligned}
$$

with $\mu>0, c_{k 1}(k=2,3,4)>0$, and $c_{1 l}(l=2,3,4)<0$. In addition, the activation function $f$ satisfies the following condition:

$\left(\mathrm{H}_{4}\right) f \in C^{3}(\mathbb{R}, \mathbb{R}), f(0)=0, f^{\prime}(0) \neq 0, f^{\prime \prime}(0)=0$ and $f^{\prime \prime \prime}(0) \neq 0$.

For instance, the nonlinear activation function commonly used in the studies and applications on neural network given by $f(u)=\tanh (u)$ posses the above property. Adopting the same notations as in Sections 3 and 4, for system (5.1), we have

$$
\alpha_{k 1}=c_{k 1} f^{\prime}(0), \quad \alpha_{1 l}=c_{1 l} f^{\prime}(0), \quad k, l=2,3,4 .
$$

From (3.5) and (3.6), one can get that

$$
\begin{gathered}
a_{3}=4 \mu>0, \quad a_{2}=6 \mu^{2}>0, \quad a_{1}=4 \mu^{3}>0, \quad a_{0}=\mu^{4}>0, \\
b_{2}=-\left(c_{12} c_{21}+c_{13} c_{31}+c_{14} c_{41}\right) f^{\prime 2}(0)>0, \\
b_{1}=-2 \mu\left(c_{12} c_{21}+c_{13} c_{31}+c_{14} c_{41}\right) f^{\prime 2}(0)>0, \\
b_{0}=-\mu^{2}\left(c_{12} c_{21}+c_{13} c_{31}+c_{14} c_{41}\right) f^{\prime 2}(0)>0,
\end{gathered}
$$

which implies the condition $\left(\mathrm{H}_{2}\right)$ holds and $a, b, c, d$ defined by (3.6) are

$$
\begin{gathered}
a=4 \mu^{2}, \quad b=6 \mu^{4}-\left(c_{12} c_{21}+c_{13} c_{31}+c_{14} c_{41}\right)^{2} f^{\prime 4}(0), \\
c=4 \mu^{6}-2 \mu^{2}\left(c_{12} c_{21}+c_{13} c_{31}+c_{14} c_{41}\right)^{2} f^{\prime 4}(0), \\
d=\mu^{8}-\mu^{4}\left(c_{12} c_{21}+c_{13} c_{31}+c_{14} c_{41}\right)^{2} f^{\prime 4}(0) .
\end{gathered}
$$


Therefore, we have $b>0, c>0, d<0$ provided that

$$
\mu^{4}<\left(c_{12} c_{21}+c_{13} c_{31}+c_{14} c_{41}\right)^{2} f^{\prime 4}(0)<2 \mu^{4} .
$$

Thus, $d h(z) / d z>0$ for all $z>0$, that is, $h(z)$ is strictly monotonous increasing on $[0, \infty)$. Noticing that $d<0$, it follows that $h(z)$ has only a positive root, say $z_{0}$. Let $\omega_{0}=\sqrt{z_{0}}$, $\tau=\tau_{1}+\tau_{2}$. From (2.16) and (5.3), we have

$$
\tau_{j}=\frac{1}{\omega_{0}}\left\{\arccos \left(\frac{\mu^{2}-\omega_{0}^{2}}{\left(c_{12} c_{21}+c_{13} c_{31}+c_{14} c_{41}\right) f^{\prime 2}(0)}\right)+2 j \pi\right\}, \quad j=0,1, \ldots
$$

By Theorem 3.1, we can get the following result.

Theorem 5.1. Suppose that the condition $\left(\mathrm{H}_{4}\right)$ and (5.5) hold and $\tau_{j}$ is defined by (5.6).

(i) When $\tau \in\left[0, \tau_{0}\right)$, all roots of the corresponding characteristic equation of (5.1) have negative real parts. Meanwhile, when $\tau=\tau_{0}$, the corresponding characteristic equation of (5.1) has only a pair of simple purely imaginary roots $\pm i \omega_{0}$, and all other roots have negative real parts. However, when $\tau>\tau_{0}$, the corresponding characteristic equation of (5.1) has at least one root with positive real part.

(ii) The zero steady state of system (5.1) is asymptotically stable when $\tau \in\left[0, \tau_{0}\right)$, and unstable when $\tau>\tau_{0}$.

(iii) System (5.1) undergoes a Hopf bifurcation at the zero steady state when $\tau=\tau_{j}, j=$ $0,1,2, \ldots$

Theorem 5.2. Assume the conditions of Theorem 5.1 hold, and $\tau_{j}$ is defined by (5.6). Then, the direction of Hopf bifurcation and stability of the bifurcating periodic solutions at $\tau_{j}$ $(j=0,1,2, \ldots)$ are determined by sign of $f^{\prime \prime \prime}(0) / f^{\prime}(0)$. More specifically, if $f^{\prime \prime \prime}(0) / f^{\prime}(0)<$ $0(>0)$, then the Hopf bifurcation is supercritical (subcritical) and the bifurcating periodic solutions are orbitally asymptotically stable (unstable) on the center manifold.

Proof. For system (5.1), from the assumption $\left(\mathrm{H}_{4}\right)$, we know that

$$
\begin{array}{ccc}
k_{1}=\frac{c_{21} f^{\prime \prime}(0)}{2 !}=0, & k_{2}=\frac{c_{21} f^{\prime \prime \prime}(0)}{3 !}, & k_{3}=\frac{c_{31} f^{\prime \prime}(0)}{2 !}=0, \\
k_{4}=\frac{c_{31} f^{\prime \prime \prime}(0)}{3 !}, & k_{5}=\frac{c_{41} f^{\prime \prime}(0)}{2 !}=0, & k_{6}=\frac{c_{41} f^{\prime \prime \prime}(0)}{3 !}, \\
l_{1}=\frac{c_{12} f^{\prime \prime}(0)}{2 !}=0, & l_{2}=\frac{c_{12} f^{\prime \prime \prime}(0)}{3 !}, & m_{1}=\frac{c_{13} f^{\prime \prime}(0)}{2 !}=0, \\
m_{2}=\frac{c_{13} f^{\prime \prime \prime}(0)}{3 !}, & n_{1}=\frac{c_{14} f^{\prime \prime}(0)}{2 !}=0, & n_{2}=\frac{c_{14} f^{\prime \prime \prime}(0)}{3 !}, \\
\alpha=\frac{c_{12} f^{\prime}(0)}{\mu+i \omega_{0}}, & \beta=\frac{c_{13} f^{\prime}(0)}{\mu+i \omega_{0}}, & \gamma=\frac{c_{14} f^{\prime}(0)}{\mu+i \omega_{0}}, \\
\alpha^{*}=\frac{c_{21} f^{\prime}(0)}{\mu-i \omega_{0}} e^{i \omega_{0} \tau_{j}}, & \beta^{*}=\frac{c_{31} f^{\prime}(0)}{\mu-i \omega_{0}} e^{i \omega_{0} \tau_{j}}, & \gamma^{*}=\frac{c_{41} f^{\prime}(0)}{\mu-i \omega_{0}} e^{i \omega_{0} \tau_{j}} .
\end{array}
$$


From (5.7) and (4.30), we can observe

$$
\begin{gathered}
g_{20}=g_{11}=g_{02}=0 \\
g_{21}=6 \bar{Q} \tau_{j}\left[\left(k_{2} \alpha^{2} \bar{\alpha}+k_{4} \beta^{2} \bar{\beta}+k_{6} \gamma^{2} \bar{\gamma}\right) e^{-i \omega_{0} \tau_{j}}+l_{2} \overline{\alpha^{*}}+m_{2} \overline{\beta^{*}}+n_{2} \overline{\gamma^{*}}\right] \\
=\frac{\left[c_{12} c_{21}\left(|\alpha|^{2}+1\right)+c_{13} c_{31}\left(|\beta|^{2}+1\right)+c_{14} c_{41}\left(|\gamma|^{2}+1\right)\right]}{\mu+i \omega_{0}} \times \bar{Q} \tau_{j} f^{\prime}(0) f^{\prime \prime \prime}(0) e^{-i \omega_{0} \tau_{j}} .
\end{gathered}
$$

From the discussion in Section 4, we know that

$$
\left(\mu+i \omega_{0}\right)^{2}=\frac{1}{3}\left(\alpha_{12} \alpha_{21}+\alpha_{13} \alpha_{31}+\alpha_{14} \alpha_{41}\right) e^{-i \omega_{0} \tau_{j}} .
$$

Therefore, we have

$$
\begin{aligned}
\bar{Q} & =\left[e^{i \omega_{0} \tau_{j}}+\frac{\alpha_{12} \alpha_{21}+\alpha_{13} \alpha_{31}+\alpha_{14} \alpha_{41}}{\left(\mu+i \omega_{0}\right)^{2}}+\frac{\left(\alpha_{12} \alpha_{21}+\alpha_{13} \alpha_{31}+\alpha_{14} \alpha_{41}\right) \tau_{j}}{\mu+i \omega_{0}}\right]^{-1} e^{i \omega_{0} \tau_{j}} \\
& =\frac{\left(\mu+i \omega_{0}\right)^{2} e^{i \omega_{0} \tau_{j}}}{\left(\mu+i \omega_{0}\right)^{2} e^{i \omega_{0} \tau_{j}}+\left(\alpha_{12} \alpha_{21}+\alpha_{13} \alpha_{31}+\alpha_{14} \alpha_{41}\right)\left[1+\left(\mu+i \omega_{0}\right) \tau_{j}\right]} \\
& =\frac{3\left(\mu+i \omega_{0}\right)^{2} e^{i \omega_{0} \tau_{j}}}{\left(c_{12} c_{21}+c_{13} c_{31}+c_{14} c_{41}\right)\left(4+3 \mu \tau_{j}+i 3 \omega_{0} \tau_{j}\right) f^{\prime 2}(0)} .
\end{aligned}
$$

Substituting (5.10) into (5.8), one can get

$$
\begin{aligned}
g_{21}= & 3 \tau_{j} \frac{f^{\prime \prime \prime}(0)}{f^{\prime}(0)} \frac{\mu\left(4+3 \mu \tau_{j}\right)+3 \omega_{0}^{2} \tau_{j}+4 \omega_{0} i}{\left(c_{12} c_{21}+c_{13} c_{31}+c_{14} c_{41}\right)\left[\left(4+3 \mu \tau_{j}\right)^{2}+9 \omega_{0}^{2}\right]} \\
& \times\left[c_{12} c_{21}\left(|\alpha|^{2}+1\right)+c_{13} c_{31}\left(|\beta|^{2}+1\right)+c_{14} c_{41}\left(|\gamma|^{2}+1\right)\right] .
\end{aligned}
$$

Noticing that $g_{20}=g_{11}=g_{02}=0$ and from (4.58), we have

$$
\begin{aligned}
\operatorname{Re}\left\{c_{1}(0)\right\}= & \frac{1}{2} \operatorname{Re}\left\{g_{21}\right\} \\
= & 3 \tau_{j} \frac{f^{\prime \prime \prime}(0)}{2 f^{\prime}(0)} \frac{\mu\left(4+3 \mu \tau_{j}\right)+3 \omega_{0}^{2} \tau_{j}}{\left(c_{12} c_{21}+c_{13} c_{31}+c_{14} c_{41}\right)\left[\left(4+3 \mu \tau_{j}\right)^{2}+9 \omega_{0}^{2}\right]} \\
& \times\left[c_{12} c_{21}\left(|\alpha|^{2}+1\right)+c_{13} c_{31}\left(|\beta|^{2}+1\right)+c_{14} c_{41}\left(|\gamma|^{2}+1\right)\right]
\end{aligned}
$$


In addition, when $\mu^{4}<\left(c_{12} c_{21}+c_{13} c_{31}+c_{14} c_{41}\right)^{2} f^{\prime 4}(0)<2 \mu^{4}$, we know that $a>0, b>0$, $c>0$, it follows that $d h(z) / d z>0$ for all $z \geq 0$. Therefore, we have $\operatorname{Re}\left\{\lambda^{\prime}\left(\tau_{j}\right)\right\}>0$. Noticing that $\mu, \omega_{0}, \tau_{j}>0(j=0,1,2, \ldots), a_{k 1}>0(k=2,3,4)$, and $a_{1 l}<0(l=2,3,4)$, we can easily obtain

$$
\begin{gathered}
\varrho_{2}=-\frac{\operatorname{Re}\left\{c_{1}(0)\right\}}{\operatorname{Re}\left\{\lambda^{\prime}\left(\tau_{j}\right)\right\}}>0(<0) \quad \text { if } \frac{f^{\prime \prime \prime}(0)}{f^{\prime}(0)}<0(>0), \\
\varsigma_{2}=2 \operatorname{Re}\left\{c_{1}(0)\right\}<(>0) \quad \text { if } \frac{f^{\prime \prime \prime}(0)}{f^{\prime}(0)}<0(>0) .
\end{gathered}
$$

By the general result of Hassard et al. [10], the conclusion of the theorem follows.

5.2. Numerical simulations. In this section, we give numerical simulations supporting our theoretical analysis. As a example, we consider the following system:

$$
\begin{gathered}
\dot{x}_{1}(t)=-2 x_{1}(t)+2 \tanh \left(x_{2}\left(t-\tau_{2}\right)\right)+\tanh \left(x_{3}\left(t-\tau_{2}\right)\right)+\tanh \left(x_{4}\left(t-\tau_{2}\right)\right), \\
\dot{x}_{2}(t)=-2 x_{2}(t)-\tanh \left(x_{1}\left(t-\tau_{1}\right)\right), \\
\dot{x}_{3}(t)=-2 x_{3}(t)-2 \tanh \left(x_{1}\left(t-\tau_{1}\right)\right), \\
\dot{x}_{4}(t)=-2 x_{4}(t)-\tanh \left(x_{1}\left(t-\tau_{1}\right)\right),
\end{gathered}
$$

which has a equilibrium $(0,0,0,0)$. It follows from (5.4) that $a=16, b=71, c=56, d=$ -144 since $f^{\prime}(0)=\tanh ^{\prime}(0)=1$. Thus, in this case $h(z)=z^{4}+16 z^{3}+71 z^{2}+56 z-144$, it is easily to see that equation $h(z)=0$ has only a positive root $z_{0}=1$, and so $\omega_{0}=1$. From (5.6), we have

$$
\tau_{j}=\arccos \left(-\frac{3}{5}\right)+2 j \pi, \quad j=0,1, \ldots
$$

where $\tau_{j}=\tau_{1 j}+\tau_{2 j}$. From Theorem 5.1, we know that the zero equilibrium of system (5.14) is asymptotically stable when $\tau_{1}+\tau_{2} \in[0,2.2143)$, this fact is illustrated by the numerical simulation in Figures 5.1-5.2 with $\tau_{1}=1.2, \tau_{2}=0.8$.

When $\tau_{1}+\tau_{2}$ is increased to the critical value 2.2143 , the origin losses its stability and Hopf bifurcation occurs. In addition, since $f^{\prime \prime \prime}(0)=\tanh ^{\prime \prime \prime}(0)=-1 / 4$, it follows from Theorem 5.2 that the Hopf bifurcation is supercritical and the projection of the bifurcating periodic solution on the center manifold is asymptotically stable, that is, the bifurcating periodic solution is orbitally asymptotically stable. In addition, all roots of (2.5) with $\tau=\tau_{0}=2.2143$, except $\pm i$, have negative real parts. Thus, the center manifold theory implies that the stability of the periodic solutions projected in the center manifold coincide with the stability of the periodic solutions in the whole phase space, this property is depicted in the numerical simulation Figures 5.3, 5.4, and 5.5 with $\tau_{1}=1.2$, $\tau_{2}=1.3$. 


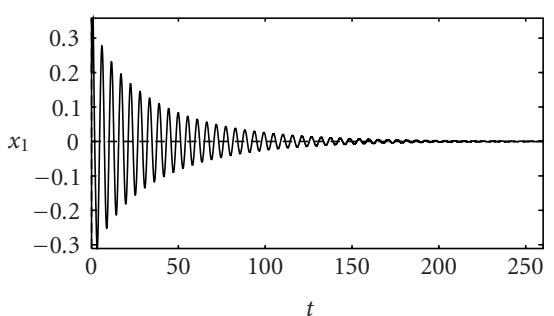

(a)

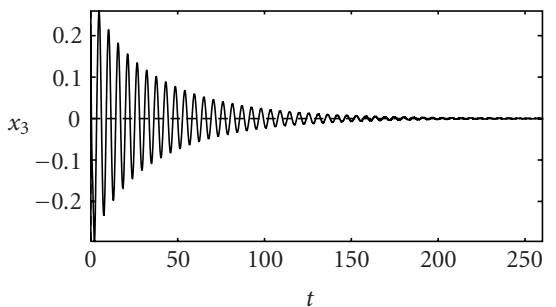

(c)

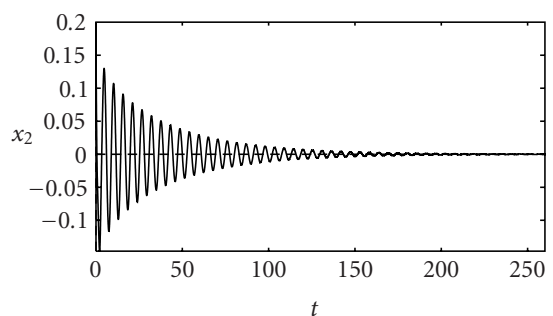

(b)

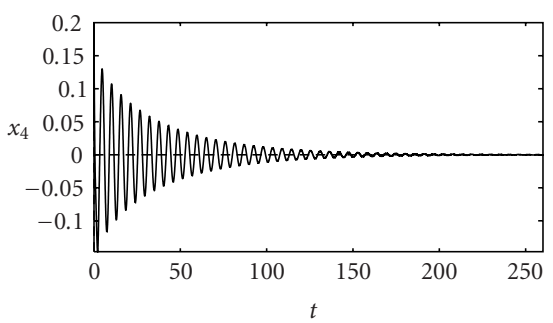

(d)

Figure 5.1. The trajectories graphs of system (5.14) with $\tau_{1}=1.2, \tau_{2}=0.8$, and initial data $x_{1}(t)=$ $x_{2}(t)=x_{3}(t)=x_{4}(t)=0.3, t \in[-1.2,0]$.

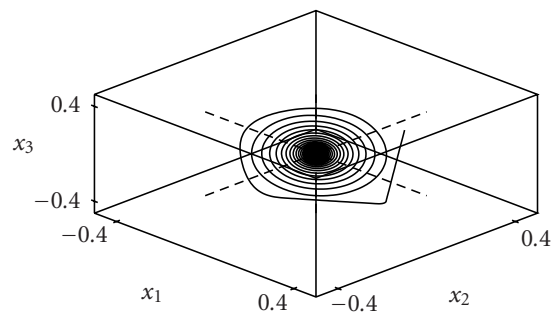

(a)

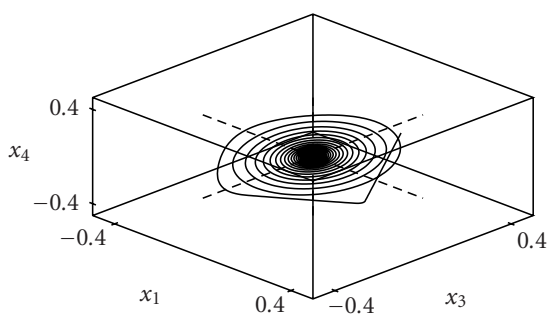

(c)

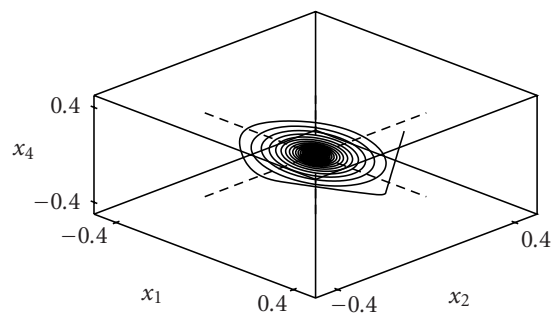

(b)

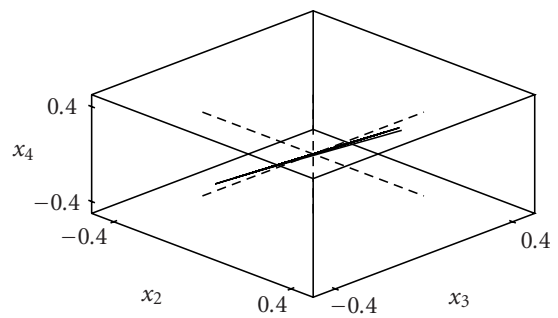

(d)

Figure 5.2. The projections in the space with dimension 3 of the orbit of system (5.14) with $\tau_{1}=1.2$, $\tau_{2}=0.8$, and initial data $x_{1}(t)=x_{2}(t)=x_{3}(t)=x_{4}(t)=0.3, t \in[-1.2,0]$. 
A BAM neural network with multiple delays

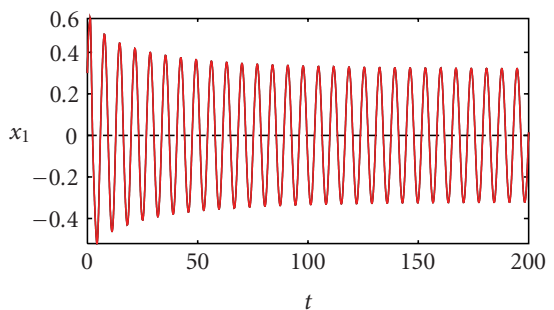

(a)

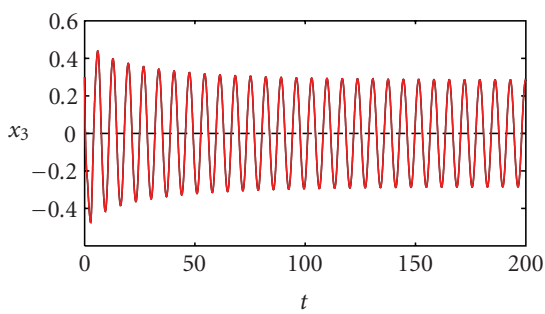

(c)

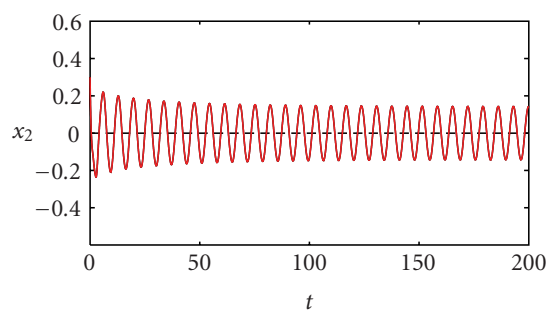

(b)

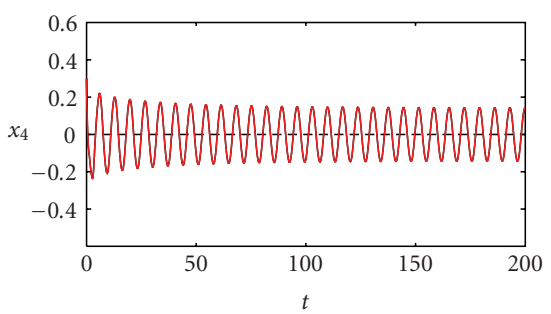

(d)

Figure 5.3. The trajectories graphs of system (5.14) with $\tau_{1}=1.2, \tau_{2}=1.3$, and initial data $x_{1}(t)=$ $x_{2}(t)=x_{3}(t)=x_{4}(t)=0.3, t \in[-1.3,0]$.

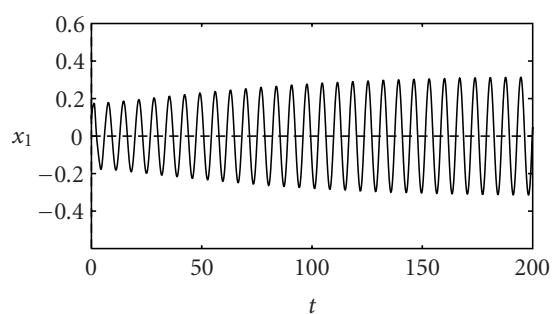

(a)

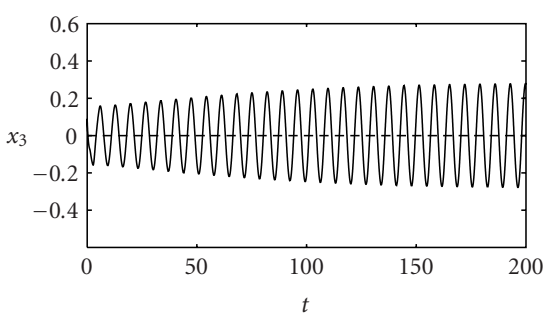

(c)

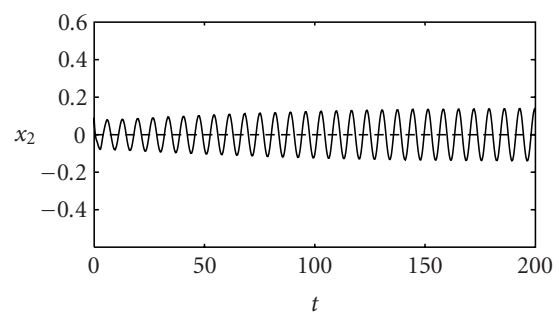

(b)

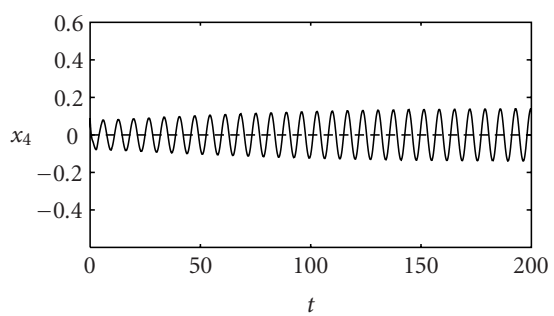

(d)

Figure 5.4. The trajectories graphs of system (5.14) with $\tau_{1}=1.2, \tau_{2}=1.3$, and initial data $x_{1}(t)=$ $x_{2}(t)=x_{3}(t)=x_{4}(t)=0.09, t \in[-1.3,0]$. 


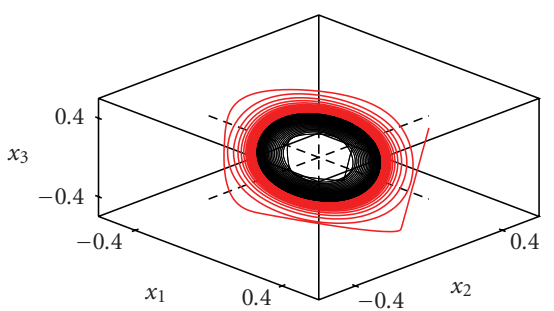

- Initial data $=0.3$

- Initial data $=0.09$

(a)

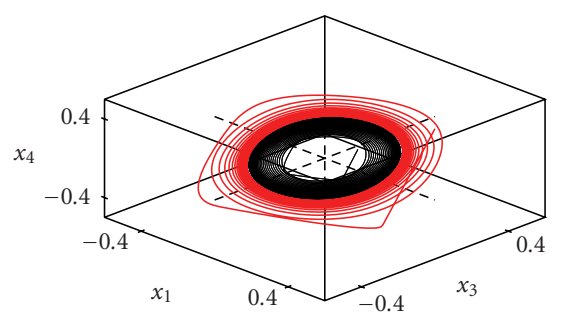

$\begin{aligned} \text { Initial data } & =0.3 \\ \text { Initial data } & =0.09\end{aligned}$

(c)

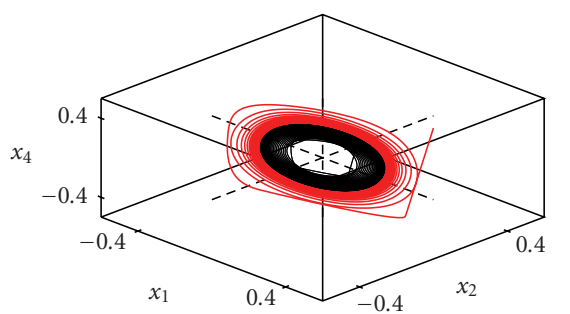

- Initial data $=0.3$

- Initial data $=0.09$

(b)

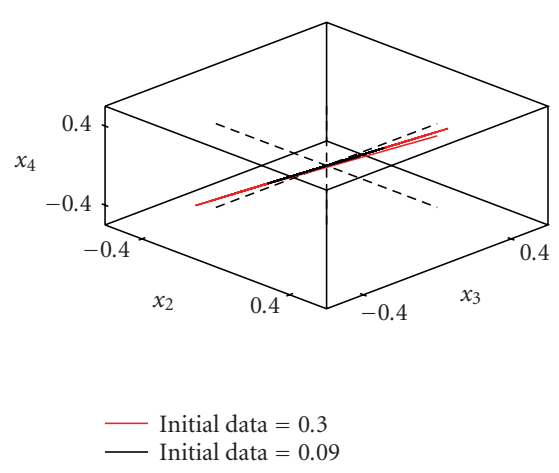

(d)

Figure 5.5. The projections in three-dimensional spaces of the orbit of system (5.14) with $\tau_{1}=1.2$, $\tau_{2}=1.3$, and initial data $x_{1}(t)=x_{2}(t)=x_{3}(t)=x_{4}(t)=0.3$, and $0.09, t \in[-1.3,0]$.

\section{Acknowledgment}

The second author was supported by the NNSF of China and the Teaching and Research Award Program for Outstanding Young Teachers in Higher Education Institutions of Ministry of Education of China.

\section{References}

[1] K. L. Babcock and R. M. Westervelt, Dynamics of simple electronic neural networks, Physica D. Nonlinear Phenomena 28 (1987), no. 3, 305-316.

[2] P. Baldi and A. F. Atiya, How delays affect neural dynamics and learning, IEEE Transactions on Neural Networks 5 (1994), no. 4, 612-621.

[3] J. Bélair, S. A. Campbell, and P. van den Driessche, Frustration, stability, and delay-induced oscillations in a neural network model, SIAM Journal on Applied Mathematics 56 (1996), no. 1, 245-255. 
[4] S. A. Campbell, S. Ruan, and J. Wei, Qualitative analysis of a neural network model with multiple time delays, International Journal of Bifurcation and Chaos in Applied Sciences and Engineering 9 (1999), no. 8, 1585-1595.

[5] F. Giannakopoulos and A. Zapp, Bifurcations in a planar system of differential delay equations modeling neural activity, Physica D. Nonlinear Phenomena 159 (2001), no. 3-4, 215-232.

[6] K. Gopalsamy and X.-Z. He, Delay-independent stability in bidirectional associative memory networks, IEEE Transactions on Neural Networks 5 (1994), no. 6, 998-1002.

[7] K. Gopalsamy and I. Leung, Delay induced periodicity in a neural netlet of excitation and inhibition, Physica D. Nonlinear Phenomena 89 (1996), no. 3-4, 395-426.

[8] J. K. Hale, Theory of Functional Differential Equations, 2nd ed., Applied Mathematical Sciences, vol. 3, Springer, New York, 1977.

[9] J. K. Hale, E. F. Infante, and F.-S. P. Tsen, Stability in linear delay equations, Journal of Mathematical Analysis and Applications 105 (1985), no. 2, 533-555.

[10] B. D. Hassard, N. D. Kazarinoff, and Y. H. Wan, Theory and Applications of Hopf Bifurcation, London Mathematical Society Lecture Note Series, vol. 41, Cambridge University Press, Cambridge, 1981.

[11] J. J. Hopfield, Neurons with graded response have collective computational properties like those of two-state neurons, Proceedings of the National Academy of Sciences of the United States of America 81 (1984), no. 10, 3088-3092.

[12] X. Li and J. Wei, On the zeros of a fourth degree exponential polynomial with applications to a neural network model with delays, Chaos, Solitons and Fractals 26 (2005), no. 2, 519-526.

[13] Z. Liu and R. Yuan, Stability and bifurcation in a harmonic oscillator with delays, Chaos, Solitons and Fractals 23 (2005), no. 2, 551-562.

[14] C. M. Marcus and R. M. Westervelt, Stability of analog neural networks with delay, Physical Review. A. Third Series 39 (1989), no. 1, 347-359.

[15] S. Mohamad, Global exponential stability in continuous-time and discrete-time delayed bidirectional neural networks, Physica D. Nonlinear Phenomena 159 (2001), no. 3-4, 233-251.

[16] L. Olien and J. Bélair, Bifurcations, stability, and monotonicity properties of a delayed neural network model, Physica D. Nonlinear Phenomena 102 (1997), no. 3-4, 349-363.

[17] S. Ruan and J. Wei, On the zeros of a third degree exponential polynomial with applications to a delayed model for the control of testosterone secretion, IMA journal of Mathematics Applied in Medicine and Biology. 18 (2001), no. 1, 41-52.

[18] Y. Song, M. Han, and J. Wei, Stability and Hopf bifurcation analysis on a simplified BAM neural network with delays, Physica D. Nonlinear Phenomena 200 (2005), no. 3-4, 185-204.

[19] L. Wang and X. Zou, Hopf bifurcation in bidirectional associative memory neural networks with delays: analysis and computation, Journal of Computational and Applied Mathematics 167 (2004), no. $1,73-90$.

[20] J. Wei and S. Ruan, Stability and bifurcation in a neural network model with two delays, Physica D. Nonlinear Phenomena 130 (1999), no. 3-4, 255-272.

[21] J. Wei and M. G. Velarde, Bifurcation analysis and existence of periodic solutions in a simple neural network with delays, Chaos. An Interdisciplinary Journal of Nonlinear Science 14 (2004), no. 3, 940-953.

[22] J. Wu, Introduction to Neural Dynamics and Signal Transmission Delay, de Gruyter Series in Nonlinear Analysis and Applications, vol. 6, Walter de Gruyter, Berlin, 2001. 
[23] F.-Y. Zhang and W.-T. Li, Global stability of delayed Hopfield neural networks under dynamical thresholds, Discrete Dynamics in Nature and Society. An International Multidisciplinary Research and Review Journal 2005 (2005), no. 1, 1-17.

Xiang-Ping Yan: School of Mathematics and Statistics, Lanzhou University, Lanzhou 730000, China Current address: School of Mathematics, Physics and Software Engineering,

Lanzhou Jiaotong University, Lanzhou 730070, China

E-mail address: yanxp@mail.lajtu.cn

Wan-Tong Li: School of Mathematics, Physics and Software Engineering, Lanzhou Jiaotong University, Lanzhou 730070, China

E-mail address: wtli@lzu.edu.cn 


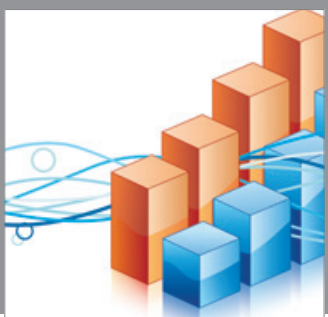

Advances in

Operations Research

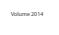

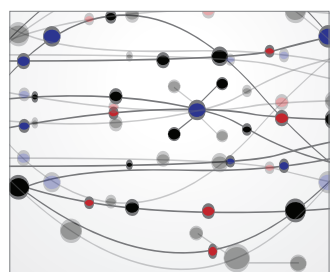

\section{The Scientific} World Journal
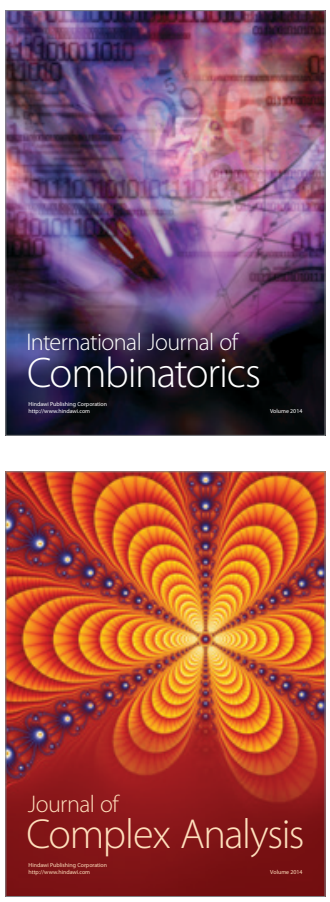

International Journal of

Mathematics and

Mathematical

Sciences
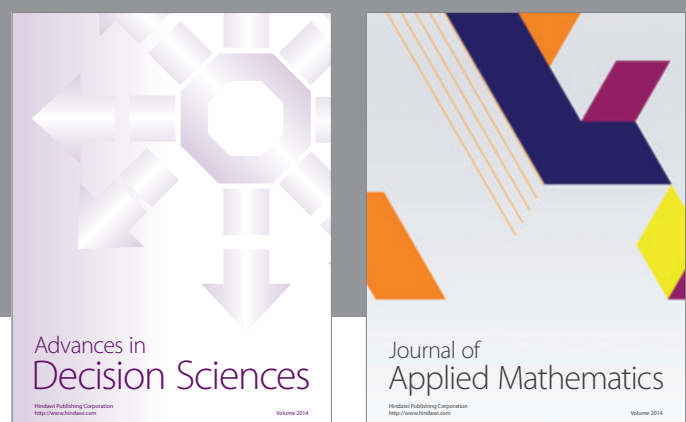

Journal of

Applied Mathematics
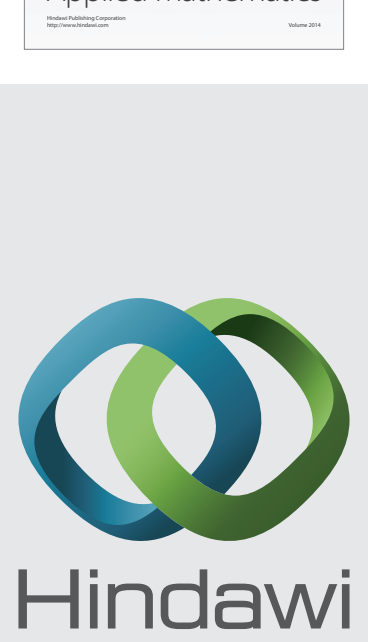

Submit your manuscripts at http://www.hindawi.com
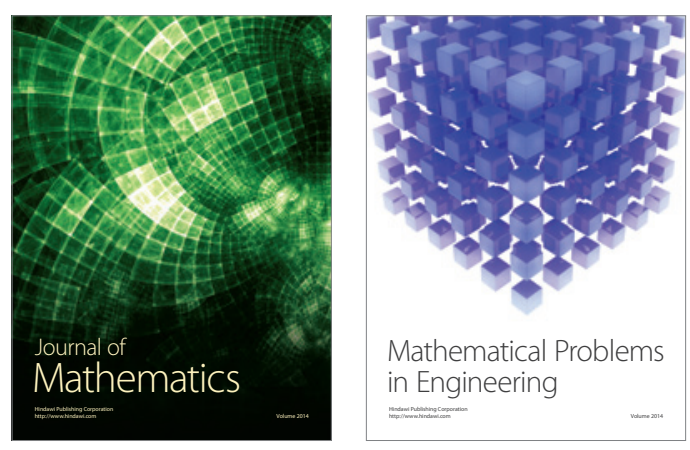

Mathematical Problems in Engineering
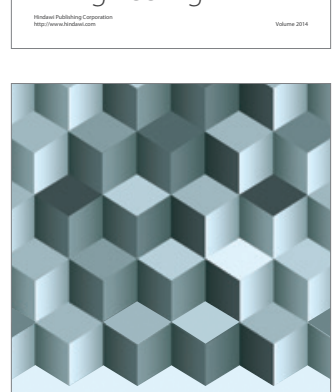

Journal of

Function Spaces
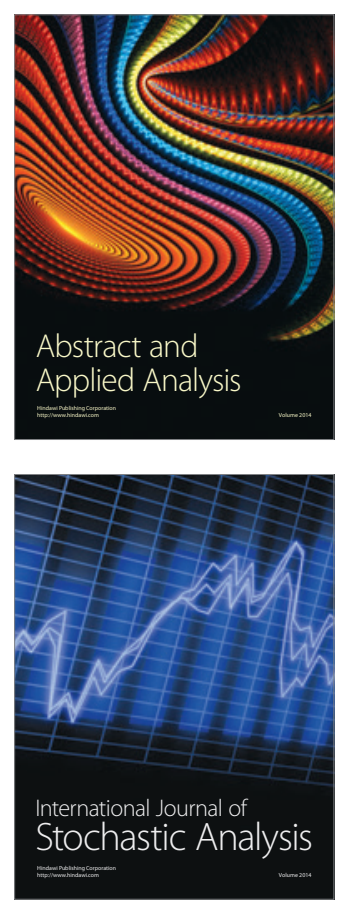

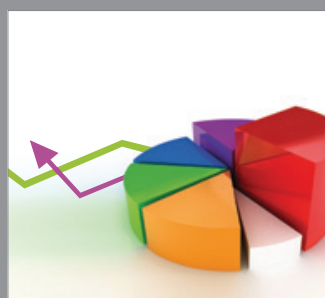

ournal of

Probability and Statistics

Promensencen
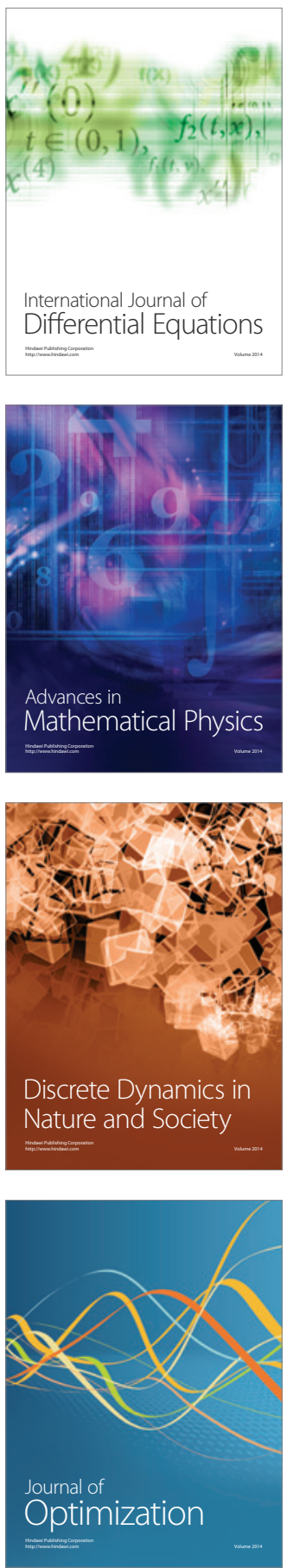